\title{
DISCRETE BALAYAGE AND BOUNDARY SANDPILE
}

\author{
HAYK ALEKSANYAN AND HENRIK SHAHGHOLIAN
}

\begin{abstract}
We introduce a new lattice growth model, which we call boundary sandpile. The model amounts to potential-theoretic redistribution of a given initial mass on $\mathbb{Z}^{d}(d \geq 2)$ onto the boundary of an (a priori) unknown domain. The latter evolves through sandpile dynamics, and has the property that the mass on the boundary is forced to stay below a prescribed threshold. Since finding the domain is part of the problem, the redistribution process is a discrete model of a free boundary problem, whose continuum limit is yet to be understood.

We prove general results concerning our model. These include canonical representation of the model in terms of the smallest super-solution among a certain class of functions, uniform Lipschitz regularity of the scaled odometer function, and hence the convergence of a subsequence of the odometer and the visited sites, discrete symmetry properties, as well as directional monotonicity of the odometer function. The latter (in part) implies the Lipschitz regularity of the free boundary of the sandpile.

As a direct application of some of the methods developed in this paper, combined with earlier results on classical Abelian sandpile, we show that the boundary of the scaling limit of Abelian sandpile is locally a Lipschitz graph.
\end{abstract}

\section{Contents}

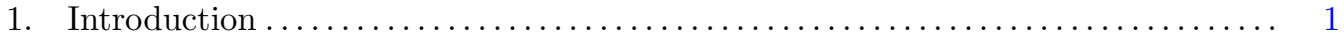

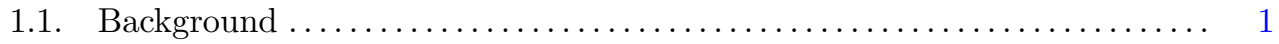

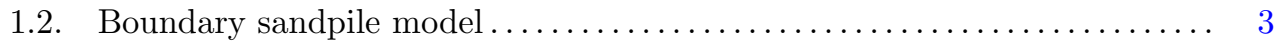

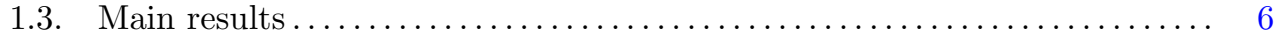

2. Well-posedness of the model $\ldots \ldots \ldots \ldots \ldots \ldots \ldots \ldots \ldots \ldots \ldots \ldots \ldots \ldots \ldots \ldots$

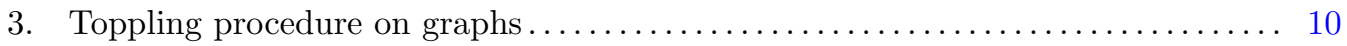

4. Discrete potential theory in the sandpile framework $\ldots \ldots \ldots \ldots \ldots \ldots \ldots \ldots \ldots \ldots 14$

4.1. The canonical domain of the model $\ldots \ldots \ldots \ldots \ldots \ldots \ldots \ldots \ldots \ldots \ldots \ldots$

4.2. Directional monotonicity for point masses $\ldots \ldots \ldots \ldots \ldots \ldots \ldots \ldots \ldots$

4.3. Applications to classical Abelian sandpile .................... 18

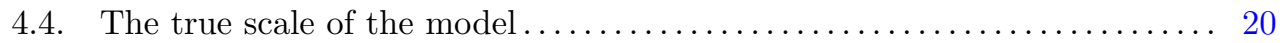

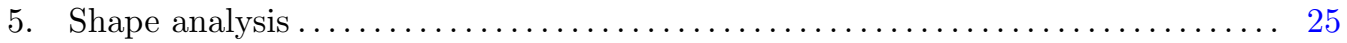

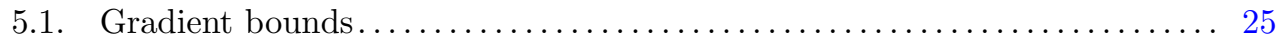

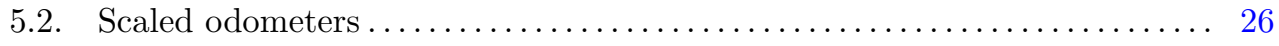

5.3. Concluding remarks and open problems $\ldots \ldots \ldots \ldots \ldots \ldots \ldots \ldots \ldots \ldots$

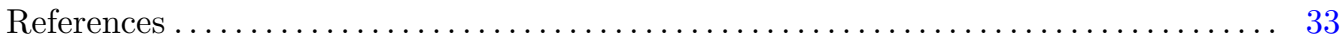

\section{INTRODUCTION}

1.1. Background. Recent years have seen a surge of modelling particle dynamics from a discrete potential theoretic perspective. The models, which usually run under

Mathematics Subject Classification (2010): 31C20, 35R35, 60J45 (31C05, 60G50, 82C41)

Key words and phrases. Boundary sandpile, balayage, lattice growth model, quadrature surface, divisible sandpile, asymptotic shape, free boundary, Abelian sandpile.

H. A. was supported by postdoctoral fellowship from Knut and Alice Wallenberg Foundation. H. Sh. was partially supported by Swedish Research Council. 
the heading aggregation models, in particular cases boil down to (harmonic/Poisson) redistribution of a given initial mass (sandpile) according to some prescribed governing rules. The most famous and well-known model is the Poincaré's Balayage, where a given initial mass distribution $\mu$ (in a given domain $D$ ) is to be redistributed (or mapped) to another distribution $\nu$ on the boundary of the domain

$$
\mathrm{Bal}: \mu \longrightarrow \nu,
$$

where $\nu$ is uniquely defined through $\mu$, and $D$. This model uses continuous amounts of mass instead of discrete ones; the latter is more common in chip firing on graphs (see [7] for instance).

A completely different model called partial-balayage $(\text { see }[15])^{1}$ aims at finding a body (domain) that is gravi-equivalent with the given initial mass. This problem, in turn, is equivalent to variational inequalities and the so-called obstacle problem. The discrete version of this problem was (probably for the first time) studied by D. Zidarov, where he performed (what is now called) a divisible sandpile model; see [31] page $108-118 .^{2}$

Levine [26], and Levine-Peres [24], [25] started a systematic study of such problems, proving, among other things, existence of scaling limit for divisible sandpiles. Although Zidarov was the first to consider such a problem, the mathematical rigour is to be attributed to Levine [26], and Levine-Peres [24], [25].

The divisible sandpile, which is of particular relevance to our paper, is a growth model on $\mathbb{Z}^{d}(d \geq 2)$ which amounts to redistribution of a given continuous mass. The redistribution of mass takes place according to a (given) simple rule: each lattice point can, and eventually must topple if it already has more than a prescribed amount of mass (sand). The amount to topple is the excess, which is divided between all the neighbouring lattice points equally or according to a governing background PDE. The scaling limit of this model, when the lattice spacing tends to 0 , and the amount of mass is scaled properly, leads to the obstacle problem in $\mathbb{R}^{d}(d \geq 2)$.

The divisible sandpile model of Zidarov, and Levine-Peres also relates to a wellknown problem in potential theory, the so-called Quadrature Domains (QD) [8]. A quadrature domain $D$ (with respect to a given measure $\mu$ ) is a domain that has the same exterior Newtonian potential (with uniform density) as that of the measure $\mu$. Hence, potential theoretically $\mu$ and $\chi_{D}$ are equivalent in the free space; i.e. outside the support of $D$ one has $U^{\mu}=U^{\chi_{D}}$, where these are the Newtonian potentials of $\mu$, respectively $\chi_{D}$. The odometer function $u$ of Levine-Peres (which represents the amount of mass emitted from each lattice site) corresponds to the difference between the above potentials (up to a normalization constant), i.e. $c_{d} u(x)=U^{\mu}-U^{\chi_{D}}$, where $u$ is $C^{1, \alpha}\left(\mathbb{R}^{d}\right)$ and $u=|\nabla u|=0$ in $\mathbb{R}^{d} \backslash D$. This, expressed differently, means that

$$
\int h(x) d \mu=\int_{D} h(x) d x
$$

for all $h$ harmonic and integrable over $D$ (see [8]).

In many other (related) free boundary value problems (known as Bernoulli type) the zero boundary gradient $|\nabla u|=0$ in the above takes a different turn, and is a prescribed (strictly) non-zero function, and the volume potential $U \chi_{D}$ in (1.2) is replaced by surface/single layer potential (of the a priori unknown domain) $U^{\chi \partial D}$. In terms of sandpile redistribution this means to find a domain $D$ such that the

\footnotetext{
${ }^{1}$ The term partial-balayage was first coined by Ognyan Kounchev as was pointed to us by Björn Gustafsson at KTH.

${ }^{2}$ Actually Zidarov (at page 109 in his book) claims to prove that the model is abelian. We have not attempted to verify the correctness of his claim.
} 
given initial mass $\mu$ in (1.1) is replaced by a prescribed mass $\nu=g(x) d \mathcal{H}_{\partial D}$ on $\partial D$. Here $\mathcal{H}_{\partial D}$ is the standard surface measure on $\partial D$, and $g$ a given function; for the simplest case $g(x) \equiv 1$ the counterpart of (1.2) would be

$$
\int h(x) d \mu=\int_{\partial D} h(x) d \mathcal{H}_{\partial D}
$$

for all $h$ harmonic on a neighbourhood of $\bar{D}$. Such domains are referred to as Quadrature Surfaces, with corresponding PDE

$$
\Delta u=-\mu+d \mathcal{H}_{\partial D}
$$

with $c_{d} u=U^{\mu}-U^{\chi \partial D}$, where $c_{d}$ is a normalization constant. This problem is well-studied in the continuum case and there is a large amount of literature concerning existence, regularity and geometric properties of both solutions and the free boundary, see [4], [16], and the references therein.

In our search to find a model for the sandpile particle dynamics to model (1.3) we came across a few models that we (naively) thought could and should be the answer to our quest. However, running numerical simulations (without any theoretical attempts) it became apparent that the models we suggested are far from the Bernoulli type free boundaries ${ }^{3}$. In particular, redistribution of an initial point mass does not converge (numerically) to a sphere, but to a shape with a boundary remotely resembling the boundary of the Abelian sandpile (see Figure ${ }^{4} 1$; cf. [28, Figure 1], or [24, Figure 2] for instance).

Notwithstanding this, our model seems to present a new alluring and fascinating phenomenon not considered earlier, neither by combinatorics nor free boundary communities. Hence the "birth" of this article.

1.2. Boundary sandpile model. Since the boundary of a set will be a prime object in the analysis of the paper, we will assume throughout the text that $d \geq 2$ to avoid uninteresting cases.

Given an initial mass $\mu$ on $\mathbb{Z}^{d}$, we want to find a model that builds a domain $D \subset \mathbb{Z}^{d}$ such that the corresponding (discrete) balayage measure $\nu$ on the boundary $\partial D$ (see (1.1)) is prescribed. Such a model seems for the moment infeasible for us. A slight variation of it asks to find a "canonical" domain such that the boundary mass $\nu$ stays below the prescribed threshold. Since larger domains (with reasonable geometry) have larger boundaries, and hence a greater amount of boundary points, we should expect that one can always find a solution to our problem by taking a very large domain containing the initial mass. This, in particular, suggests that a canonical domain should be the smallest domain among all domains with the property that the boundary mass $\nu$ is below the prescribed mass, which we shall assume to be uniform mass. ${ }^{5}$

Departing from the above point of view of canonicity one may ask whether there is a natural lattice growth model which corresponds to this minimality. ${ }^{6}$ It turns

\footnotetext{
3 Although uniqueness (in general) fails for Bernoulli type free boundary problems, it is well-known that in the case of good geometry of data (here Dirac mass) and (partial) smoothness of the free boundary one obtains uniqueness of the solution. In particular a domain $D$ admitting quadrature identity (1.3), in continuum case, should be a sphere.

${ }^{4}$ This of course is far from a reasonable shape for a Bernoulli free boundary!

${ }^{5}$ Solving the above problem in $\mathbb{R}^{d}$ results in a so-called Bernoulli free boundary problem, where the Green's potential $G_{\mu, D}$ of the mass $\mu$, in the sought domain $D$, has boundary gradient $\left|\nabla G_{\mu, D}\right|=1$, in case of uniform distribution, i.e. $\nu=d \mathcal{H}_{\partial D}$.

${ }^{6}$ It should be remarked that the divisible sandpile related to the obstacle problem corresponds to smallest (super)solution to $\Delta w \leq 1$ with $w \geq G_{-\mu}$.
} 

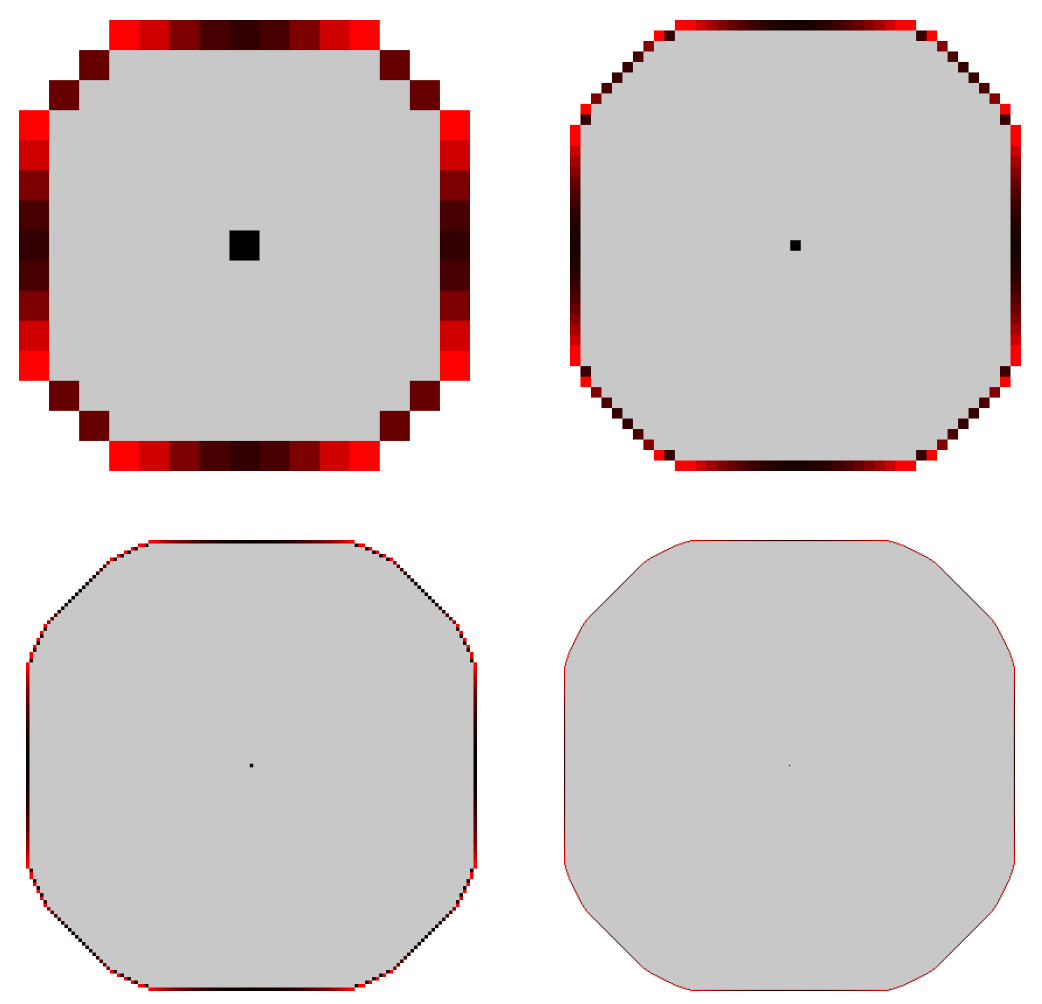

FIGURE 1. From top left to bottom right are the sets of visited sites on $\mathbb{Z}^{2}$ of the 2-dimensional BS with initial mass $n$ concentrated at the origin and equal to $10^{3}, 10^{4}, 10^{5}$ and $10^{6}$ respectively, where in all cases the boundary capacity is set to $n^{1 / 2}$. The gray region is the set where the odometer is harmonic. On the boundary, darker colors indicate higher concentration of mass, and the black dot at the center represents the origin.

out that there actually is a model which seemingly is more complicated than the divisible sandpile model described above. To introduce our model, which we call boundary sandpile, we need a few formal definitions ${ }^{7}$.

1.2.1. Constants. Through the text various letters $c, C, c_{1}, \ldots$ will stand for constants which may change from formulae to formula. We use lower case $c$ to denote small constants, and upper case $C$ for large constants.

1.2.2. Lattice notation. Call two lattice points $x, y \in \mathbb{Z}^{d}$ neighbours and write $x \sim y$ if $\|x-y\|_{l^{1}}=1$, where $l^{1}$-norm of $x \in \mathbb{R}^{d}$ is the sum of absolute values of its coordinates. Clearly $x \sim y$ iff $x=y \pm e_{i}$ for some $1 \leq i \leq d$ where $e_{i}$ is the $i$-th vector of the standard basis of $\mathbb{R}^{d}$. For any non-empty set $V \subset \mathbb{Z}^{d}$, this concept of lattice adjacency induces a natural graph $G$ having vertices on $V$. We call such $G$ the lattice graph of $V$.

Next, for a set $V \subset \mathbb{Z}^{d}$ define

$$
\partial V:=\{x \in V: \exists y \sim x \text { such that } y \notin V\} \text { and } \stackrel{\circ}{V}=V \backslash \partial V,
$$

and call them respectively the boundary of $V$ and the interior of $V$. For the convenience of the exposition we have chosen to include the boundary into the set.

\footnotetext{
${ }^{7}$ Although this is a generic name, as there are obviously many other ways to topple particles/mass onto the boundary (see Remark 1.1), we think it might be more convenient without any further specification with descriptive names to use the term Boundary Sandpile.
} 
For $h>0$ recall the definition of the discrete Laplace operator denoted by $\Delta^{h}$ and acting on a function $u: h \mathbb{Z}^{d} \rightarrow \mathbb{R}$ by

$$
\Delta^{h} u(x)=\frac{1}{2 d h^{2}} \sum_{y \sim_{h} x}[u(y)-u(x)], \quad x \in h \mathbb{Z}^{d},
$$

where $y \sim_{h} x$, as for $h=1$, means that $y$ is a neighbour of $x$ in the lattice $h \mathbb{Z}^{d}$. Throughout the text, when $h=1$, i.e. on a unit scale lattice, we will write $\Delta$ for $\Delta^{1}$ unless explicitly stated otherwise.

For $R>0$ we set

$$
Z_{R}=\left\{\xi \in \mathbb{Z}^{d}:|\xi| \leq R\right\} \cup\left\{x \in \mathbb{Z}^{d}: \exists \xi \in \mathbb{Z}^{d} \text { s.t. } \xi \sim x \text { and }|\xi| \leq R<|x|\right\}
$$

for the closed discrete ball of radius $R$ including its 1-neighbourhood. It is clear from the definition that $x \in \mathbb{Z}^{d}$ is from the interior of $Z_{R}$ iff $|x| \leq R$.

1.2.3. Definition of the process. Let now $\mu_{0}$ be a given (mass) distribution on $\mathbb{Z}^{d}$, i.e. a non-negative function supported on finitely many sites of $\mathbb{Z}^{d}$. Fix also a threshold $\kappa_{0}>0$. For each integer $k \geq 0$ we inductively construct a sequence of sets $V_{k}$, mass distributions $\mu_{k}$, and functions $u_{k}$ as follows. Start with $V_{0}=\operatorname{supp} \mu_{0}$ and $u_{0}=0$. For an integer time $k \geq 0$ a particular site $x \in \mathbb{Z}^{d}$ is called unstable if either of the following holds:

(a) $x \in \partial V_{k}$ and $\mu_{k}(x)>\kappa_{0}$,

(b) $x \in \stackrel{\circ}{V_{k}}$ and $\mu_{k}(x)>0$.

Otherwise a site is called stable. We call the number $\kappa_{0}$ the (boundary) capacity of the model and refer to $V_{k}$ as the set of visited sites at time $k$.

Any unstable site can topple by distributing all its mass equally among its $2 d$ lattice neighbours. More precisely, for each $k \geq 0$ we choose an unstable site $x \in V_{k}$ and define $V_{k+1}=V_{k} \cup\left\{y \in \mathbb{Z}^{d}: y \sim x\right\}$,

$$
\mu_{k+1}(y)= \begin{cases}0, & \text { if } y=x, \\ \mu_{k}(y)+\frac{1}{2 d} \mu_{k}(x), & \text { if } y \sim x, \\ \mu_{k}(y), & \text { otherwise }\end{cases}
$$

and $u_{k+1}(y)=u_{k}(y)+\mu_{k}(y) \delta_{0}(y-x), y \in \mathbb{Z}^{d}$, where $\delta_{0}$ is the Kronecker delta symbol at the origin, i.e. $\delta_{0}(x)$ equals 1 if $x=0$ and is zero otherwise for $x \in \mathbb{Z}^{d}$. We call $u_{k}$ the odometer function at time $k$. For the sake of convenience, we do allow the toppling to be applied to a stable site, as an identity operator, i.e. if at time $k$ a toppling is applied to a stable site $x$, then we set $V_{k+1}=V_{k}, u_{k+1}=u_{k}$ and $\mu_{k+1}=\mu_{k}$. We say that toppling $x$ is legal, if $x$ is unstable. If for some $k$ there are no unstable sites, the process is terminated. We call this model boundary sandpile (BS) and denote by $\operatorname{BS}\left(\mu_{0}, \kappa_{0}\right)$, where $\mu_{0}$ is the initial distribution, and $\kappa_{0}$ is the boundary capacity of the model.

It is clear that the triple $\left(V_{k}, \mu_{k}, u_{k}\right)_{k=1}^{\infty}$ may depend on the choice of the unstable sites, i.e. the toppling sequence. Later on we will see that for a suitable class of toppling sequences stable configurations exist and are identical (see Propositions 2.1 and 2.3). Observe that from the definition of discrete Laplacian and (1.5) we easily see that for each $k \geq 0$ one has

$$
\Delta u_{k}(x)=\mu_{k}(x)-\mu_{0}(x), \quad x \in \mathbb{Z}^{d},
$$

i.e. the Laplacian of $u_{k}$ represents the net gain of mass for a site $x$ at time $k$.

We further define a few concepts needed for our analysis. For a $\operatorname{BS}\left(\mu_{0}, \kappa_{0}\right)$, and a (toppling) sequence $T=\left\{x^{(k)}\right\}_{k=1}^{\infty} \subset \mathbb{Z}^{d}$, we say that $T$ is stabilizing if there 
exists a distribution $\mu$ such that $\mu_{k}(x) \rightarrow \mu(x)$ as $k \rightarrow \infty$ for any $x \in \mathbb{Z}^{d}$. We call $T$ infinitive on a set $V \subset \mathbb{Z}^{d}$ if every $x \in V$ appears in the sequence $T$ infinitely often. If the set on which $T$ is infinitive is not specified, then it is assumed to be the entire $\mathbb{Z}^{d}$.

1.3. Main results. Our main results concern general qualitative analysis of the boundary sandpile model, introduced in this paper. For any initial mass distribution we prove well-posedness of the model (Propositions 2.1 and 2.3), and canonical representation of the model in terms of the smallest super-solution among a certain class of functions (Theorem 4.2). Specifying our analysis for point masses and using this minimality of the model, we show directional monotonicity of the odometer function (Theorem 4.5). We then determine the reasonable size of the boundary capacity (subsection 4.4.1) and estimate the growth rate of the model in Lemma 4.12. We prove a uniform Lipschitz bound for the scaled odometer function in Proposition 5.2. Using these results obtained in discrete setting, in the final part of the paper, Section 5 we study the scaling limit of the model in continuum space. In particular, we show that (along a subsequence) the scaled odometers, and hence the visited sites, converge to a continuum limit (Theorem 5.3 parts (i) and (iii)). We also prove that the free boundary of (any) scaling limit of the model is locally a Lipschitz graph (Theorem 5.3 part (v)).

In subsection 4.3 we apply our methods developed for boundary sandpile model to classical Abelian sandpile. Most notably, we show that the boundary of the scaling limit of Abelian sandpile corresponding to initial configuration of chips at a single vertex, has Lipschitz boundary.

Remark 1.1. It is worthwhile to remark that the boundary sandpile described above can be represented slightly differently, using discrete partial derivatives. Indeed, our model generates a set $V \subset \mathbb{Z}^{d}$ whose Green's function $u$ (corresponding to the given initial distribution of mass) satisfies

$$
\frac{1}{2 d} \sum_{i=1}^{d}\left(\left|\partial_{i}^{+} u(x)\right|+\left|\partial_{i}^{-} u(x)\right|\right)-\kappa_{0} \leq 0 \quad \text { on } \partial V
$$

for a given $\kappa_{0}$, where $\partial_{i}^{ \pm} u(x)=u\left(x \pm e_{i}\right)-u(x)$. In light of this observation one may consider a wider class of problems in terms of general prescribed boundary mass given by $F\left(x, \partial_{1}^{ \pm} u, \ldots, \partial_{d}^{ \pm} u\right)$, where $F$ defined on $\mathbb{Z}^{d} \times \mathbb{R}_{+}^{2 d}$ has to satisfy some properties (ellipticity and other), yet to be found. The methods developed in this paper seem to have a good chance to go through for at least "nice enough" function F. This aspect we shall leave for interested reader to explore.

\section{WELL-POSEDNESS OF THE MODEL}

In this section we prove two basic properties of the boundary sandpile model. Namely, that the model needs to visit a finite number of sites in $\mathbb{Z}^{d}$ to reach a stable state, and that the final stable configuration is independent of the toppling sequence. For the proofs we will use some ideas from [24, Lemma 3.1] and [25, Lemma 3.1].

Proposition 2.1. (Existence of odometer function) For a given $\operatorname{BS}\left(\mu_{0}, \kappa_{0}\right)$ any infinitive toppling sequence $T$ is stabilizing.

Proof. Let $n:=\sum_{x \in \mathbb{Z}^{d}} \mu_{0}(x)$ be the total mass of the sandpile, and set $V_{0}=$ $\operatorname{supp} \mu_{0}$. For each $k \in \mathbb{N}$ let $V_{k}$ be the set of visited sites after invoking $k$-th toppling in $T$. Let also $u_{k}$ and $\mu_{k}$ be respectively the odometer function and the distribution of mass after the $k$-th site in $T$ has toppled. 

has

We first show that for any $k \in \mathbb{N}$ and for each $x \in \partial V_{k}$ satisfying $\mu_{0}(x)=0$ one has

$$
\mu_{k}(x)>\frac{1}{2 d} \kappa_{0} .
$$

Indeed, let $1 \leq i \leq k$ be the smallest integer such that $x \in V_{i} \backslash V_{i-1}$, and let $y \in V_{i-1}$ be the neighbour of $x$ that topples at time $i$. Since $y \in V_{i-1}$ and $y \sim x \notin V_{i-1}$ we have $y \in \partial V_{i-1}$, but as toppling of $y$ is legal (since it is producing a previously unvisited site $x$ ), we must have $\mu_{i-1}(y)>\kappa_{0}$. Consequently we get $\mu_{i}(x)>\frac{1}{2 d} \kappa_{0}$, and since $x$ did not topple at steps $i+1, \ldots, k$, as otherwise $x$ will be in the interior of $V_{k}$, we get $\mu_{k}(x)=\mu_{i}(x)$ and hence (2.1). Comparing (2.1) with the total mass $n$, for any $k \geq 0$ we get

$$
\left|\partial V_{k} \backslash V_{0}\right| \leq 2 d \frac{n}{\kappa_{0}}
$$

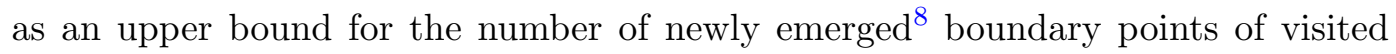
sites at any time of the process.

Now assume that $V_{k}$ is connected as a lattice graph. Fix $1 \leq \alpha \leq d$ and let $a, b \in \partial V_{k}$ be such that $a_{\alpha}=\min _{x \in \partial V_{k}} x_{\alpha}$ and $b_{\alpha}=\max _{x \in \partial V_{k}} x_{\alpha}$. For each integer $a_{\alpha} \leq$ $c \leq b_{\alpha}$ consider the hyperplane $H_{c}=\left\{x \in \mathbb{R}^{d}: x_{\alpha}=c\right\}$. It is clear that $H_{c} \cap V_{k}$ contains at least one element of $\partial V_{k}$ and these points are pairwise different for integer $c \in\left[a_{\alpha}, b_{\alpha}\right]$. By $(2.2)$ one has

$$
b_{\alpha}-a_{\alpha} \leq 2 d \frac{n}{\kappa_{0}}+\left|V_{0}\right|
$$

As $1 \leq \alpha \leq d$ is arbitrary, for any $x \in V_{k}$ we obtain

$$
\max _{1 \leq \alpha \leq d} \max _{y \in V_{0}}\left|(x-y)_{\alpha}\right| \leq 2 d \frac{n}{\kappa_{0}}+\left|V_{0}\right|
$$

hence the process occupies a finite region of $\mathbb{Z}^{d}$ independently of ${ }^{9} k$. It is clear that assuming connectedness of $V_{k}$ resulted in no loss of generality, as we can employ the scanning procedure by hyperplanes on each connected component of the lattice graph of $V_{k}$.

Since the toppling is infinitive, for each $k \geq 1$ all unstable sites on $\partial V_{k}$ will topple eventually, by so extending the set of visited sites. But (2.3) shows that after finite number of topplings, the boundary of visited sites will become stable and will remain so throughout. Precisely, there is $k \geq 1$ and a set $V \subset \mathbb{Z}^{d}$ such that $V_{i}=V$ for all $i \geq k$. By (1.6) for any integer $k \geq 0$ we have

$$
\Delta u_{k}=\mu_{k}-\mu_{0} \text { in } \mathbb{Z}^{d} \text {. }
$$

The r.h.s. of the last expression, as well as each $u_{k}$ are supported in $V$ for all integer $k \geq 0$. We thus have that the functions $x \mapsto \Delta u_{k}(x)$, independently of $k \in \mathbb{N}$, are uniformly bounded on $\mathbb{Z}^{d}$, and have compact supports. Take any $x \in \partial V$, then $u_{k}(x)=0$ and $\mu_{k}(x) \leq \kappa_{0}$ in view of the stability of $V$. Hence

$$
\frac{1}{2 d} \sum_{y \sim x} u_{k}(y)=\Delta u_{k}(x)=\mu_{k}(x)-\mu_{0}(x) \leq \kappa_{0},
$$

\footnotetext{
${ }^{8}$ Here one can only estimate the number of boundary points of the visited sites which were not initially in the support of $\mu_{0}$. The simple reason is that in general $\mu_{0}$ may have, say, arbitrarily large number of isolated points with small mass, which may never be visited again in the course of the process.

${ }^{9}$ Later on in Lemma 4.12 we will obtain more precise bounds for the set of visited sites. However, the bound in Lemma 4.12 uses a priori boundedness of the set of visited sites.
} 
which shows that $\left\{u_{k}(y)\right\}_{k=1}^{\infty}$ is uniformly bounded for any $y \sim x$. This uniform bound on $u_{k}$ propagates into each connected component of the lattice graph of $V$. Hence we get $u_{k}(x) \leq C$ for any $x \in \mathbb{Z}^{d}$ and any $k \geq 1$ with some constant $C=C(d, n)$.

Since for each $x \in \mathbb{Z}^{d}$, the sequence $\left\{u_{k}(x)\right\}_{k=1}^{\infty}$ is increasing and bounded above, it has a finite limit which we denote by $u(x)$. Clearly $u$ is non-negative and compactly supported. It also follows that the l.h.s. of (2.4) must have a limit everywhere in $\mathbb{Z}^{d}$. Passing to the limit in $(2.4)$ as $k$ goes to infinity, we get $\Delta u=\mu-\mu_{0}$ in $\mathbb{Z}^{d}$ where by $\mu$ we denote the limit distribution of $\mu_{k}$.

To complete the proof it is left to observe that the final distribution $\mu$ is stable. As we saw above, $\mu$ is stable on $\partial V$, hence it remains to show that $\mu=0$ in the interior of $V$. Fix any $x \in \stackrel{\circ}{V}$, since $T$ is infinitive we get $\mu_{k}(x)=0$ for infinitely many $k$. But as $\mu(x)=\lim _{k \rightarrow \infty} \mu_{k}(x)$ and the limit exists, we obtain $\mu(x)=0$ completing the proof of the proposition.

Remark 2.2. It is clear from the proof of Proposition 2.1 that one can require the toppling to be infinitive only on the set of visited sites. We do not do so as the final domain is not known a priori.

We call the function $u$ constructed in the proof of Proposition 2.1 the odometer and the distribution $\mu$ the final configuration corresponding to the given toppling sequence.

Proposition 2.3. (Abelian property) For a given $\mathrm{BS}\left(\mu_{0}, \kappa_{0}\right)$ and any two infinitive toppling sequences $T_{1}$ and $T_{2}$, one has $u_{1}=u_{2}$ for the corresponding odometer functions.

Proof. As in Proposition 2.1 by $n$ denote the total mass of the system. In view of Proposition 2.1 each $T_{i}$ is stabilizing and has well-defined odometer function, call it $u_{i}$. We will prove that $u_{2}(x) \geq u_{1}(x)$ for any $x \in \mathbb{Z}^{d}$, which by symmetry will imply the desired result.

For $i=1,2$ by $u_{i, k}$ denote the odometer function and by $\mu_{i, k}$ the distribution corresponding to the sequence $T_{i}$ after the $k$-th toppling occurs. Let $T_{1}=\left\{x^{(k)}\right\}_{k=1}^{\infty}$, we now show that

$$
u_{2}\left(x^{(k)}\right) \geq u_{1, k}\left(x^{(k)}\right), \quad k=1,2, \ldots .
$$

Observe that (2.5) is enough for our purpose since the site $x^{(k)}$ appears infinitely often in $T_{1}$ and $u_{1, k}$ converges to $u_{1}$ pointwise as $k$ tends to infinity. Thus, in what follows we prove (2.5) which we will do by induction on $k$.

When $k=1,(2.5)$ is trivially true. Now assume that it holds for any time $1 \leq i<k$. We divide the analysis into two cases.

Case 1. Toppling $x^{(k)}$ in $T_{1}$ at time $k$ is not a legal move.

In this case we have

$$
u_{1, k}\left(x^{(k)}\right)=u_{1, k-1}\left(x^{(k)}\right) .
$$

So, if $x^{(k)}$ has never toppled in $T_{1}$ prior to time $k$, then $u_{1, k-1}\left(x^{(k)}\right)=0$ and we are done. Otherwise, let $i \leq k-1$ be the last time $x^{(k)}$ has toppled in $T_{1}$. We thus have $x^{(i)}=x^{(k)}$, hence, using the inductive hypothesis, we conclude

$$
u_{2}\left(x^{(k)}\right)=u_{2}\left(x^{(i)}\right) \geq u_{1, i}\left(x^{(i)}\right)=u_{1, k-1}\left(x^{(i)}\right)=u_{1, k}\left(x^{(k)}\right),
$$


where the last equality follows from (2.6). This completes the induction step for the case of a non-legal move.

Case 2. Toppling $x^{(k)}$ in $T_{1}$ at time $k$ is legal.

We first show that for any $x \neq x^{(k)}$ one has

$$
u_{2}(x) \geq u_{1, k}(x) \text {. }
$$

There are two possible sub-cases, either $x$ was never toppled in $T_{1}$ up to time $k$, which implies $u_{1, k}(x)=0$ and we get (2.7), or $x$ was toppled at some time before $k$. In the latter case let $i \leq k$ be the last time $x$ has toppled prior to time $k$. Observe that $i<k$ since $x \neq x^{(\bar{k})}$. Then we have

$$
u_{1, k}(x)=u_{1, i}(x) \leq u_{2}(x),
$$

where the second inequality follows by inductive hypothesis. We thus have proved (2.7) for all $x \neq x^{(k)}$. Consider the following inequality

$$
\mu_{2}\left(x^{(k)}\right) \leq \mu_{1, k}\left(x^{(k)}\right) .
$$

To complete the induction step suppose for a moment that (2.8) holds true. Then

$$
\begin{aligned}
\frac{1}{2 d} \sum_{y \sim x^{(k)}} u_{2}(y)-u_{2}\left(x^{(k)}\right)=\Delta u_{2}\left(x^{(k)}\right)=\mu_{2}\left(x^{(k)}\right)-\mu_{0}\left(x^{(k)}\right) \stackrel{(2.8)}{\leq} \\
\mu_{1, k}\left(x^{(k)}\right)-\mu_{0}\left(x^{(k)}\right)=\Delta u_{1, k}\left(x^{(k)}\right)=\frac{1}{2 d} \sum_{y \sim x^{(k)}} u_{1, k}(y)-u_{1, k}\left(x^{(k)}\right) .
\end{aligned}
$$

Rearranging the first and the last terms leads to

$$
u_{2}\left(x^{(k)}\right)-u_{1, k}\left(x^{(k)}\right) \geq \frac{1}{2 d} \sum_{y \sim x^{(k)}}\left(u_{2}(y)-u_{1, k}(y)\right) \geq 0,
$$

where the last inequality is due to (2.7). This completes the induction. Thereby, to finish the proof of the proposition, we need to verify (2.8) which we do next.

To prove (2.8) we will assume that $x^{(k)}$ is on the boundary of the visited sites generated by $T_{2}$, call it $V_{2} \subset \mathbb{Z}^{d}$, as otherwise the l.h.s. of (2.8) vanishes and the inequality is trivial. Recall, that we are in Case 2, hence there are two possible reasons for $x^{(k)}$ to topple in $T_{1}$ at time $k$. Namely,

(a) $x^{(k)}$ was on the boundary of visited sites of $T_{1}$ at time $k-1$ and had mass strictly greater than $\kappa_{0}$,

(b) $x^{(k)}$ was in the interior of visited sites of $T_{1}$ at time $k-1$ and had positive mass.

We show that either of the cases leads to a contradiction. Assume (a), then

$$
\begin{aligned}
\kappa_{0}<\mu_{1, k-1}\left(x^{(k)}\right) & =\Delta u_{1, k-1}\left(x^{(k)}\right)-\mu_{0}\left(x^{(k)}\right) & & \\
& =\frac{1}{2 d} \sum_{y \sim x^{(k)}} u_{1, k-1}(y)-\mu_{0}\left(x^{(k)}\right) & & \left(\text { since } u_{1, k-1}\left(x^{(k)}\right)=0\right) \\
& \leq \frac{1}{2 d} \sum_{y \sim x^{(k)}} u_{2}(y)-\mu_{0}\left(x^{(k)}\right) & & (\text { from }(2.7)) \\
& =\Delta u_{2}\left(x^{(k)}\right)-\mu_{0}\left(x^{(k)}\right) & & \left(\text { since } u_{2}\left(x^{(k)}\right)=0\right) \\
& =\mu_{2}\left(x^{(k)}\right)-\mu_{0}\left(x^{(k)}\right) \leq \kappa_{0} & & \left(\text { as } T_{2} \text { is stabilizing) },\right.
\end{aligned}
$$

which is a contradiction. 
Now consider the case when $x^{(k)}$ topples according to (b). Recall that $x^{(k)} \in \partial V_{2}$. This implies that $x^{(k)}$ cannot topple in $T_{1}$ prior to time $k$. Indeed, if $x^{(k)}$ topples for some $i<k$, then $u_{1, i}\left(x^{(k)}\right)>0$, but by inductive hypothesis we have $0=u_{2}\left(x^{(k)}\right) \geq$ $u_{1, i}\left(x^{(k)}\right)>0$ which is false. Next let $i<k$ be the first time when the toppling of $T_{1}$ produces a vertex outside $V_{2}$, and let $y$ be the site that topples at time $i$. On one hand $y$ has to be on the boundary of the set visited by $T_{2}$, on the other hand, as we saw already, $y$ must be different from $x^{(k)}$. Hence by $(2.7)$ and the fact that $y \in \partial V_{2}$ we get $u_{1, i}(y) \leq u_{1, k}(y)=0$ meaning that $y$ cannot topple. We arrive at a contradiction, hence (2.8) is proved, and so is the proposition.

A simple consequence of the abelian property is the following lattice symmetries of the sandpile. Consider the set of directions

$$
\mathcal{N}=\left\{e_{i}, e_{i}+e_{j}, e_{i}-e_{j}, 1 \leq i \neq j \leq d\right\} \subset \mathbb{Z}^{d},
$$

and for a given $e \in \mathcal{N}$ let $T_{e}$ be the hyperplane through the origin with a normal vector collinear with $e$. Then, $T_{e}$ is a mirror symmetry hyperplane of the unit cube $[-1 / 2,1 / 2]^{d} \subset \mathbb{R}^{d}$, and any symmetry hyperplane of this cube is of the form $T_{e}$ for some $e \in \mathcal{N}$. Mirror reflections with respect to a hyperplane $T_{e}$ preserve the lattice $\mathbb{Z}^{d}$. These symmetries are inherited by sandpile with single source as we see next.

Corollary 2.4. (lattice symmetries of the model) Let the initial distribution $\mu_{0}$ be a point mass supported at the origin, and let $V$ be its final domain of visited sites, and $u$ be the odometer function. Then both $V$ and $u$ are symmetric with respect to any hyperplane $T_{e}$ where $e \in \mathcal{N}$.

Proof. Fix $e \in \mathcal{N}$ and for each $x \in \mathbb{Z}^{d}$ denote $[x]=\left\{x, x_{e}\right\}$, where $x_{e} \in \mathbb{Z}^{d}$ is the mirror reflection of $x$ with respect to the hyperplane $T_{e}$. Take any infinitive toppling $T=\left\{x^{(k)}\right\}_{k=1}^{\infty}$ for $\mu_{0}$ and consider its "symmetrized" version $[T]=\left\{\left[x^{(k)}\right]\right\}_{k=1}^{\infty}$, where each $x^{(k)}$ in $T$ is replaced by its corresponding 2-element class $[x]$. Clearly the new toppling sequence $[T]$ is infinitive, and hence stabilizing in view of Proposition 2.1. Notice that since $x$ and $x_{e}$ are not lattice neighbours, then the topplings of $x$ and $x_{e}$ do not interfere, and can be toppled in any order. Also, if $u_{k}$ is the odometer function after toppling both elements of $\left[x^{(k)}\right]$ in $[T]$, then it is symmetric with respect to hyperplane $T_{e}$ by construction, namely $u_{k}(x)=u_{k}\left(x_{e}\right)$ for any $x \in \mathbb{Z}^{d}$. Since the odometer $u$ corresponding to $[T]$ is the pointwise limit of $u_{k}$, we get the symmetry of the odometer and the set of visited sites with respect to $T_{e}$.

As $e \in \mathcal{N}$ was arbitrary, the abelian property of the sandpile completes the proof of the corollary.

Remark 2.5. One can see, in a similar way, that for any initial distribution $\mu_{0}$, its final configuration $V$ and odometer function $u$ inherit lattice symmetries of $\mu_{0}$. We do not formulate this rigorously, as later on we will be using the symmetry for point masses only.

\section{TOPPLING PROCEDURE ON GRAPHS}

It is apparent that our boundary sandpile process requires infinite number of toppling steps to reach a stable configuration (see Remark 3.3 below). Nonetheless, it is not hard to observe that the process can be stabilized effectively in finite number of steps by absorbing part of the topplings into certain linear operators acting on graphs. This fact might also be useful for numerical simulations. 
Let $G=(V, E)$ be a finite connected, simple (i.e. without loops and multiple edges) graph. Partition $V$ into two non-empty subsets $V_{0}$ and $V_{1}$ which we call source and sink respectively. For a given function $\mu: V_{0} \rightarrow \mathbb{R}_{+}$, in analogy with (1.5), consider the toppling transformation $T$ acting on a vertex $v \in V_{0}$ by

$$
\left(T_{v} \mu\right)(u)= \begin{cases}0, & \text { if } u=v, \\ \mu(u)+\frac{\mu(v)}{\operatorname{deg}(v)}, & \text { if } u \sim v, \\ \mu(u), & \text { otherwise }\end{cases}
$$

where $\operatorname{deg}(v)$ is the degree of the vertex $v$ in $G$, which is always positive in view of the connectedness of the graph. We also denote $\mathbb{E}[\mu]=\frac{1}{\left|V_{0}\right|} \sum_{v \in V_{0}} \mu(v)$ for the average of $\mu$. With these preparations we easily obtain the following.

Lemma 3.1. For any map $\sigma: \mathbb{N} \rightarrow V_{0}$ such that the pre-image of each $v \in V_{0}$ is infinite, for all $v \in V_{0}$ we have

$$
\lim _{N \rightarrow \infty}\left(T_{\sigma(N)} \circ \ldots \circ T_{\sigma(1)} \mu\right)(v)=0 .
$$

Proof. We will assume without loss of generality that the subgraph on $V_{0}$ is connected, as otherwise we can apply the argument that follows to each connected component of the subgraph. Set $d_{*}:=\max _{v \in V_{0}} \operatorname{deg}(v)$ and let $\rho$ be the diameter of the subgraph on $V_{0}$, i.e. the maximal length of the shortest path between any two vertices. Assume that the maximum of $\mu$ is attained at some $v \in V_{0}$, and take any shortest path from $v$ to the sink $V_{1}$ through the vertices of $V_{0}$. Let $v=v_{1} \sim \ldots \sim v_{k} \sim v^{*} \in V_{1}$ be such path where each $v_{i} \in V_{0}$. We have $k \leq \rho$ by definition. From the definition of $\sigma$ we can choose $N \in \mathbb{N}$ large enough such that the sequence $\left(v_{i}\right)_{i=1}^{k}$ is a subsequence of $(\sigma(i))_{i=1}^{N}$. But after toppling $v_{1}, \ldots, v_{k}$ the total mass of $V_{0}$ is decreased by at least $\mu\left(v_{1}\right) / d_{*}^{\rho}$, and as $\mu\left(v_{1}\right)=\max _{v \in V_{0}} \mu(v)$, we have

$$
\mathbb{E}\left[T_{\sigma(N)} \circ \ldots \circ T_{\sigma(1)} \mu\right] \leq \mathbb{E}[\mu]\left(1-\frac{1}{\left|V_{0}\right|} \frac{1}{d_{*}^{\rho}}\right) .
$$

Since the average is reduced by a constant multiple the claim follows.

Remark 3.2. By adapting the proof of Proposition 2.3 (in fact in a simpler form) one can easily show that the amount of mass each vertex of the sink receives is independent of the toppling order, as long as each source vertex is toppled infinite number of times.

Remark 3.3. Take a graph with two vertices and a single edge connecting them. Let also each of these two vertices be attached to any number of sinks (possibly none, but not simultaneously). Then clearly one has to topple each vertex infinitely often in order to entirely transfer any given mass to the sinks. Hence the requirement on each vertex to topple infinite number of times cannot be eliminated.

We will see shortly that one can avoid a straightforward application of topplings to transform mass from source to sink. By realizing this transference as a linear operator we will perform the mass transportation from source to sink effectively in one step.

Keeping the notation of Lemma 3.1 let the source be $V_{0}=\left\{v_{1}, \ldots, v_{N}\right\}$ and the mass vector be equal to $\mu_{0}=\left(m_{1}, \ldots, m_{N}\right)$. Consider the toppling $T=T_{v_{N}} \circ \ldots \circ T_{v_{1}}$ and for $k \in \mathbb{N}$ let $T^{k}$ denote the $k$-th iterate of $T$. Then by Lemma 3.1 we have that $T^{k} \mu_{0} \rightarrow 0$ as $k \rightarrow \infty$ and the goal is to determine how much mass each sink vertex 
will receive in the limit. Due to the abelian property (Remark 3.2) the amount of mass distributed to the sinks is independent of the toppling sequence, hence specifying the toppling order results in no loss of generality. To compute the final distribution of the mass on the sink we introduce matrices $M, D \in M_{N}(\mathbb{R})$ where we call $M$ the mass transformation matrix, and $D$ the mass distribution matrix. More precisely $M_{i j}$ shows the proportion of $\mu_{0}\left(v_{j}\right)$ which is present at vertex $v_{i}$ after we have applied the transformation $T$ to the graph. Namely if $T \mu_{0}$ is the new mass-vector after applying $T$, then

$$
\left[T \mu_{0}\right]\left(v_{i}\right)=M_{i 1} \mu_{0}\left(v_{1}\right)+M_{i 2} \mu_{0}\left(v_{2}\right)+\ldots+M_{i N} \mu_{0}\left(v_{N}\right) .
$$

In a similar fashion $D_{i j}$ shows the proportion of $\mu_{0}\left(v_{j}\right)$ transferred to its neighbours by $v_{i}$. Thus each sink (if any) attached to $v_{i}$ gets mass equal to

$$
D_{i 1} \mu_{0}\left(v_{1}\right)+D_{i 2} \mu_{0}\left(v_{2}\right)+\ldots+D_{i N} \mu_{0}\left(v_{N}\right) .
$$

A simple bookkeeping procedure illustrated below in Algorithm 1 allows us to compute these coefficients when we consequently topple $v_{1} \rightarrow v_{2} \rightarrow \ldots . \rightarrow v_{N}$.

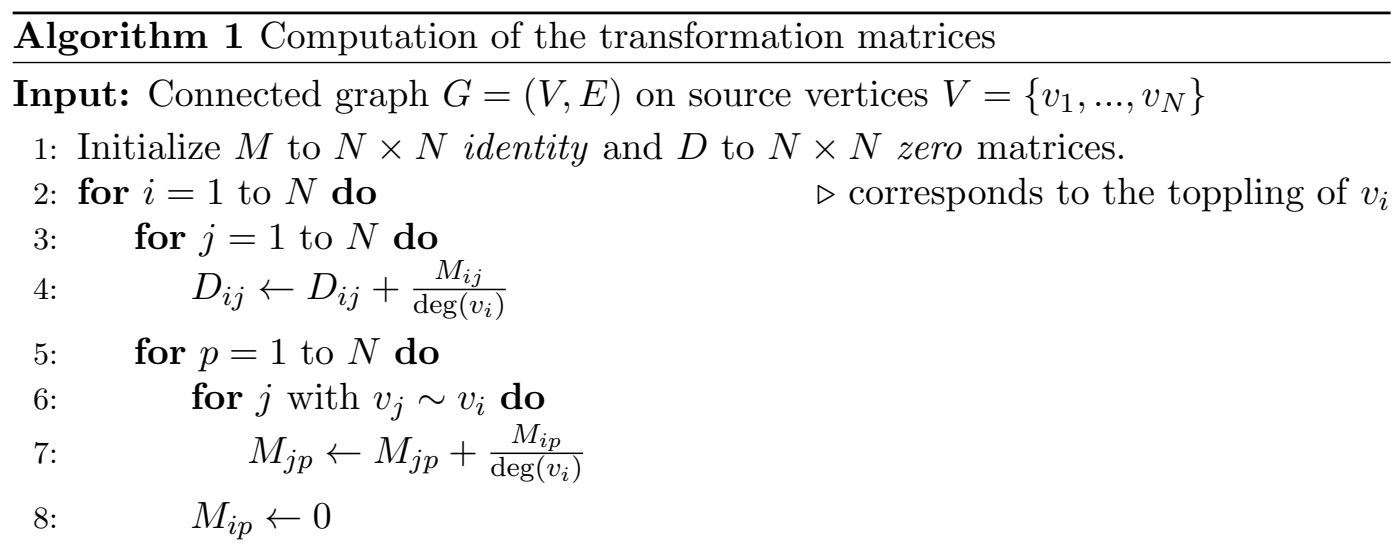

Output: Matrices $M$ and $D$

Now observe that after the toppling $T^{k}, k \in \mathbb{N}$, is applied, the mass vector distributed to sinks becomes

$$
\mu_{k}=D \mu_{0}+D\left[M \mu_{0}\right]+\ldots+D\left[M^{k-1} \mu_{0}\right]=D\left[\sum_{i=0}^{k-1} M^{i}\right] \mu_{0} \in \mathbb{R}^{N} .
$$

To sum the series in (3.3) we need the following.

Lemma 3.4. All eigenvalues of $M$ are less than 1 by absolute value.

Proof. Take any vector $\mu_{0} \in \mathbb{R}^{N}$ with all coordinates being non-negative. By virtue of (3.2) there exists an integer $k \geq 1$ and a constant $0<c<1$ such that

$$
\mathbb{E}\left[M^{k} \mu_{0}\right] \leq(1-c) \mathbb{E}\left[\mu_{0}\right]
$$

Since $M$ has non-negative entries due to construction by Algorithm 1, from the last inequality we get

$$
\left\|M^{k} \mu_{0}\right\|_{l^{1}} \leq(1-c)\left\|\mu_{0}\right\|_{l^{1}}
$$

now for any $\mu_{0} \in \mathbb{R}^{N}$. Hence, if $\mu_{0} \in \mathbb{R}^{N} \backslash\{0\}$ is an eigenvector of $M$ corresponding to an eigenvalue $\lambda$, we get

$$
\left\|M^{k} \mu_{0}\right\|_{l^{1}}=|\lambda|^{k}\left\|\mu_{0}\right\|_{l^{1}} \leq(1-c)\left\|\mu_{0}\right\|_{l^{1}}
$$

which implies $|\lambda|^{k} \leq(1-c)<1$ completing the proof of the lemma. 
Lemma 3.4 implies that the matrix Id $-M$ is invertible, where Id is the $N \times N$ identity matrix. Hence the Neumann series of $M$, as we have in (3.3), is convergent. Passing to limit in (3.3) as $k \rightarrow \infty$ demonstrates that the total mass $\mu$ distributed to the sinks equals

$$
\mathbb{R}^{N} \ni \mu=D\left[\sum_{i=0}^{\infty} M^{i}\right] \mu_{0}=D(\operatorname{Id}-M)^{-1} \mu_{0} .
$$

Observe that all mass is being transferred to the sink by Lemma 3.1, and hence there is no loss of mass. This shows that the linear operator defined by (3.4) is non-degenerate. Hence, the matrix $D$ is invertible as well. Finally, each sink vertex attached to $v_{i}$ gets mass equal to the $i$-th component of the vector

$$
\left[D(\operatorname{Id}-M)^{-1} \mu_{0}\right] \in \mathbb{R}^{N} .
$$

Based on the above discussion, we next present a simple pseudocode, which allows to stabilize the given initial distribution in finite number of steps.

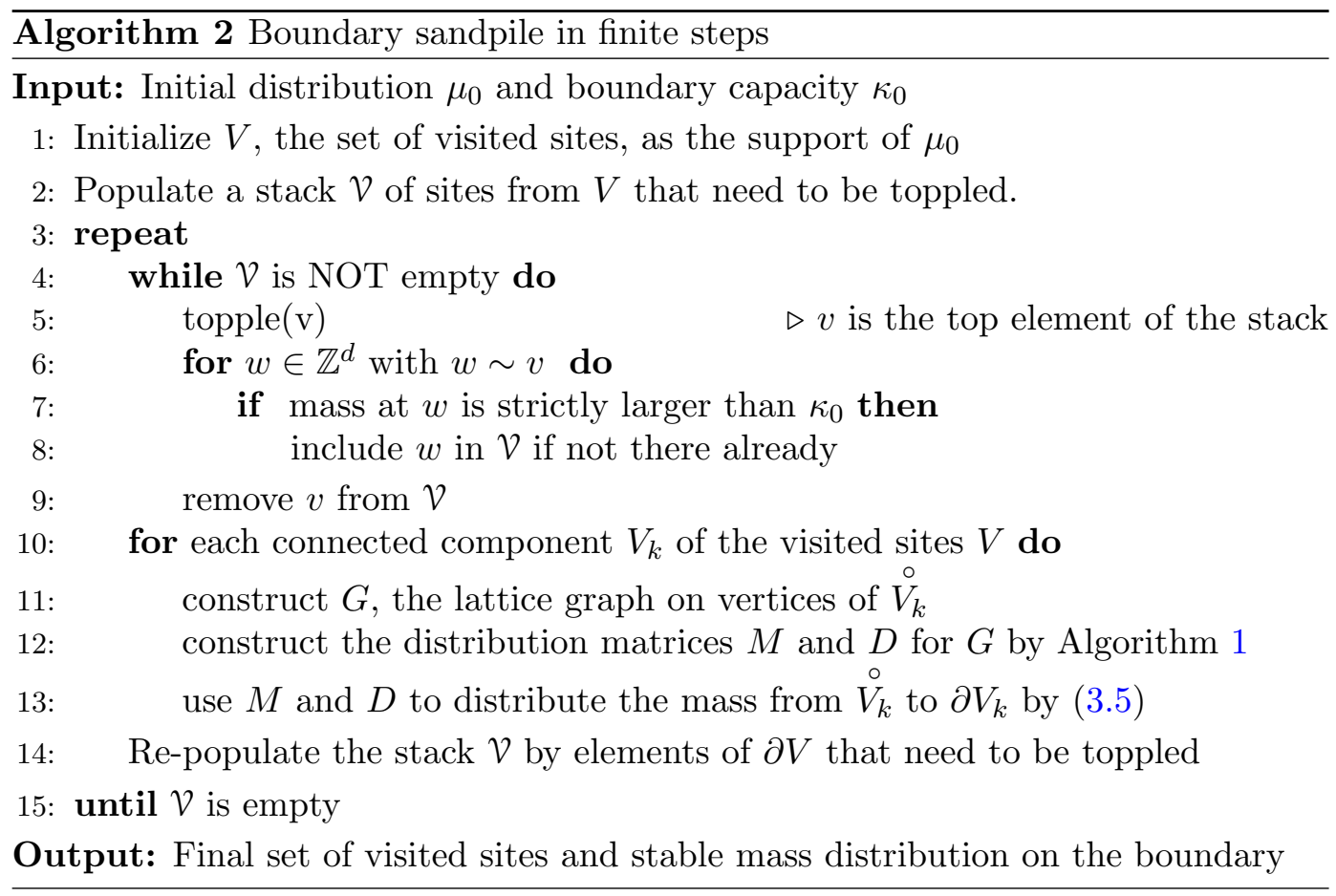

In Algorithm 2 we simply move the boundary of the set of visited sites as long as there is a possibility. Then, we transfer at once all mass from the interior onto the boundary which requires a passage to the limit in a toppling procedure. Consequently, the fact that this will produce the same final configuration as the original boundary sandpile process, is not a direct corollary of abelian property of the sandpile, but follows from Lemma 4.4. In particular, due to Lemma 4.4 each iteration of the algorithm resuming from Step 3, starts with an initial distribution $\mu$ for which the two sandpiles $\operatorname{BS}\left(\mu, \kappa_{0}\right)$ and $\operatorname{BS}\left(\mu_{0}, \kappa_{0}\right)$ produce the same final configurations. This fact, coupled with Proposition 2.1 implies that Algorithm 2 terminates in finite number of steps since the set of visited sites cannot grow to infinity ${ }^{10}$.

\footnotetext{
${ }^{10}$ We do not attempt to estimate the complexity of Algorithm 2 depending on $\mu_{0}$ and $\kappa_{0}$, however, it is interesting to observe, that the same approach can stabilize the divisible sandpile of Levine-Peres $[24]$ in finite number of steps.
} 


\section{Discrete POTENTIAL THEORY IN THE SANDPILE FRAMEWORK}

4.1. The canonical domain of the model. We start with the following.

Discrete maximum principle (DMP): Let $u, v: \mathbb{Z}^{d} \rightarrow \mathbb{R}, V \subset \mathbb{Z}^{d}$ be finite, and $\Delta u \geq \Delta v$ in the interior of $V$. Then $\max _{\partial V}(u-v) \geq \max _{V}(u-v)$.

The proof of this important statement is an easy exercise (see e.g. [21, Exercise 1.4.7]). We now show that the boundary sandpile requires the smallest possible domain in the discrete space, where it can stabilize itself.

Definition 4.1. (Stabilizing pair) For an initial distribution $\mu_{0}$ and a boundary capacity $\kappa_{0}$, we say that the pair $(V, u)$ is stabilizing for $\mathrm{BS}\left(\mu_{0}, \kappa_{0}\right)$ if the following holds:

(i) $V \subset \mathbb{Z}^{d}$ is a finite set and $\operatorname{supp} \mu_{0} \subset V$,

(ii) $u: V \rightarrow \mathbb{R}_{+}$is a function satisfying

$$
\begin{cases}\Delta u=-\mu_{0}, & \text { in } \stackrel{\circ}{V}, \\ u=0, & \text { on } \partial V, \\ \mu_{0}+\Delta u \leq \kappa_{0}, & \text { on } \partial V .\end{cases}
$$

In this setting, we will call $u$ odometer function.

Remark 4.1. For any $\mu_{0}$ and $\kappa_{0}$ let $V_{0}$ be the set of visited sites of the corresponding $\mathrm{BS}$ and $u_{0}$ be the odometer function. Then clearly the pair $\left(V_{0}, u_{0}\right)$ is stabilizing in a sense of Definition 4.2.

Theorem 4.2. (The minimality principle) Let $V_{0} \subset \mathbb{Z}^{d}$ be the set of visited sites of $\operatorname{BS}\left(\mu_{0}, \kappa_{0}\right)$. Then $V_{0}$ is the intersection of all $V \subset \mathbb{Z}^{d}$ for which there is a function $u$ such that the pair $(V, u)$ is stabilizing for $\operatorname{BS}\left(\mu_{0}, \kappa_{0}\right)$.

Proof. The set of stabilizing pairs is non-empty in view of Remark 4.1. Hence if $V_{*}$ is the intersection in the formulation of the theorem, then $V_{*}$ is finite and contains the support of $\mu_{0}$.

First, we show that for some $u_{*}$ the pair $\left(V_{*}, u_{*}\right)$ is stabilizing. To see this for $V_{*}$, it is enough to prove that for any two stabilizing pairs $\left(V_{i}, u_{i}\right), i=1,2$ the set $V:=V_{1} \cap V_{2}$ has the property. To this end, consider the function $v(x):=$ $\min \left\{u_{1}(x), u_{2}(x)\right\}$ in $V$. Clearly

$$
\partial V=\left(\partial V_{1} \cap V_{2}\right) \cup\left(\partial V_{2} \cap V_{1}\right),
$$

from which we get $v=0$ on $\partial V$. It is also clear that $\Delta v \leq-\mu_{0}$ in $\stackrel{\circ}{V}$. Next, using the fact that $v$ vanishes on $\partial V$ and is the pointwise minimum of $u_{1}$ and $u_{2}$, from (4.1) we have

$$
\Delta v+\mu_{0} \leq \kappa_{0} \text { on } \partial V .
$$

Now let $w$ be the unique solution to

$$
\Delta w=-\mu_{0} \text { in } \stackrel{\circ}{V} \text { and } \quad w=0 \text { on } \partial V .
$$

Since $w-v$ is a subsolution in the interior of $V$, from DMP we have $w \leq v$ in $V$. Combining this with the fact that both $w$ and $v$ vanish on $\partial V$ we arrive at

$$
\Delta w \leq \Delta v \text { on } \partial V \text {. }
$$

But then,

$$
\Delta w+\mu_{0} \leq \Delta v+\mu_{0} \leq \kappa_{0} \text { on } \partial V
$$


where the last inequality is due to (4.2). Hence, the pair $(V, w)$ is stabilizing and the claim for $V_{*}$ follows.

We are now in a position to show $V_{0}=V_{*}$. By definition of $V_{*}$ we have $V_{*} \subset V_{0}$. Take any toppling sequence $T=\left\{x^{(i)}\right\}_{i=1}^{\infty}$ where $x^{(i)} \in \stackrel{\circ}{*}_{*}$ for all $i \in \mathbb{N}$ and $T$ is infinitive ${ }^{11}$ on $\stackrel{\circ}{V}_{*}$. Observe that if $T$ is stabilizing for the original BS, then $V_{*}=V_{0}$ as the final configuration is unique by abelian property of the sandpile. Hence, it is left to prove that $T$ is stabilizing. Assume it is not. Invoking $T$, we get that all mass from the interior of $V_{*}$ is being redistributed onto $\partial V_{*}$ by Lemma 3.1. We have $V_{*} \subset V_{0}$, and since $T$ is not stabilizing it follows that the distribution of mass on $V_{*}$ obtained after applying $T$ is not stable. Since no mass remains in the interior of $V_{*}$ it follows that the boundary of $V_{*}$ is unstable. Let $u$ be the odometer function corresponding to the toppling $T$. Then, by definition we have

$$
\Delta u=-\mu_{0} \text { in } \stackrel{\circ}{V}_{*} \text { and } u=0 \text { on } \partial V_{*} .
$$

Let also $u_{*}$ be the odometer for which $\left(V_{*}, u_{*}\right)$ is stabilizing, whose existence was established above. By definition, $u_{*}$ satisfies (4.3) hence, due to uniqueness of solutions, we get that $u=u_{*}$. But then

$$
\Delta u+\mu_{0}=\Delta u_{*}+\mu_{0} \leq \kappa_{0} \text { on } \partial V_{*}
$$

where the inequality follows since the pair $\left(V_{*}, u_{*}\right)$ is stabilizing. This shows, that $\left(V_{*}, u\right)$ is stabilizing as well, which contradicts our assumption that the toppling $T$ was not stabilizing, and completes the proof of the theorem.

Definition 4.2. (The stabilizing pair) For a given $\mathrm{BS}\left(\mu_{0}, \kappa_{0}\right)$ we call $(V, u)$ the stabilizing pair of the sandpile if $V$ is minimal in a sense of Theorem 4.2.

Remark 4.3. An immediate reformulation of the previous theorem is the following characterization of the odometer as the smallest supersolution in a certain class of functions on $\mathbb{Z}^{d}$. For $\mathrm{BS}\left(\mu_{0}, \kappa_{0}\right)$ let $u$ be the odometer function. Then

$$
u=\inf \left\{u: \mathbb{Z}^{d} \rightarrow \mathbb{R}_{+}: \Delta u \leq-\mu_{0} \text { in } \stackrel{\circ}{\mathcal{U}} \text { and } \mu_{0}+\Delta u \leq \kappa_{0} \text { on } \partial \mathcal{U}\right\},
$$

where $\mathcal{U}:=\left\{x \in \mathbb{Z}^{d}: u(x)>0\right\} \cup \operatorname{supp} \mu_{0}$.

A useful corollary of the minimality principle, which is being used in Algorithm 2 above, is the following.

Lemma 4.4. Let $\left(V_{0}, u_{0}\right)$ be the stabilizing pair for $\mathrm{BS}\left(\mu_{0}, \kappa_{0}\right)$. Fix any $V_{1} \subset V_{0}$ such that $\operatorname{supp} \mu_{0} \subset V_{1}$ and let $u_{0 \rightarrow 1}$ be the solution to

$$
\Delta u_{0 \rightarrow 1}=-\mu_{0} \text { in } \stackrel{\circ}{V_{1}} \text { and } u_{0 \rightarrow 1}=0 \text { on } \partial V_{1} .
$$

Then the final configurations of $\mathrm{BS}\left(\mu_{0}, \kappa_{0}\right)$ and $\mathrm{BS}\left(\Delta u_{0 \rightarrow 1}+\mu_{0}, \kappa_{0}\right)$ are identical.

The lemma states that if we balayage $\mu_{0}$ to $\partial V_{1}$ first, and then balayage the resulting measure from $\partial V_{1}$ to $\partial V_{0}$, the boundary measure produced on $\partial V_{0}$ will be the same as if we had balayaged $\mu_{0}$ on $\partial V_{0}$. This is obvious from a potential theoretic and balayage perspective. It is however not straightforward from a BS dynamic point of view.

Proof of Lemma 4.4. Set $\mu_{1}:=\Delta u_{0 \rightarrow 1}+\mu_{0}$, which, by the definition of $u_{0 \rightarrow 1}$, is a distribution supported on $\partial V_{1}$. Let $\left(V_{2}, u_{2}\right)$ be the stabilizing pair for $\operatorname{BS}\left(\mu_{1}, \kappa_{0}\right)$.

\footnotetext{
${ }^{11}$ Observe that $\stackrel{\circ}{V}_{*}$ might be empty, in which case $T$ is empty as well, that is no toppling occurs. This corresponds to the case, when the initial distribution $\mu_{0}$ is already stable.
} 
The aim is to show that $V_{2}=V_{0}$ and that the final configurations for $\mathrm{BS}\left(\mu_{0}, \kappa_{0}\right)$ and $\operatorname{BS}\left(\mu_{1}, \kappa_{0}\right)$ coincide. The latter is equivalent to equality $\mu_{0}+\Delta u_{0}=\mu_{1}+\Delta u_{2}$ on $\mathbb{Z}^{d}$, which in turn is equivalent to

$$
\Delta u_{0}=\Delta\left(u_{2}+u_{0 \rightarrow 1}\right) \text { on } \mathbb{Z}^{d}
$$

which we will prove below.

We have

$$
\Delta u_{2}=-\mu_{1} \text { in } \stackrel{\circ}{V}_{2} \text { and } u_{2}=0 \text { on } \partial V_{2},
$$

as well as $\mu_{2}:=\mu_{1}+\Delta u_{2} \leq \kappa_{0}$ on $\partial V_{2}$. Clearly $V_{1} \subset V_{2}$ therefore $u_{0 \rightarrow 1}+u_{2}=0$ on $\partial V_{2}$. We also have

$$
\Delta\left(u_{0 \rightarrow 1}+u_{2}\right)=\mu_{1}-\mu_{0}+\mu_{2}-\mu_{1}=\mu_{2}-\mu_{0} \text { in } V_{2},
$$

hence the pair $\left(V_{2}, u_{0 \rightarrow 1}+u_{2}\right)$ is stabilizing for $\mathrm{BS}\left(\mu_{0}, \kappa_{0}\right)$. As a consequence of Theorem 4.2 we obtain $V_{0} \subset V_{2}$. We now prove the reverse inclusion. Since $V_{1} \subset V_{0}$, it follows that $\mu_{1}$ is supported in $V_{0}$. Hence, we may define $u_{1 \rightarrow 0}$ as the unique solution of

$$
\Delta u_{1 \rightarrow 0}=-\mu_{1} \text { in } \stackrel{\circ}{V_{0}} \text { and } u_{1 \rightarrow 0}=0 \text { on } \partial V_{0} .
$$

Since $V_{1} \subset V_{0}$ it follows that $u_{0 \rightarrow 1}+u_{1 \rightarrow 0}=0$ on $\partial V_{0}$. This implies that the function $w:=u_{0}-\left(u_{0 \rightarrow 1}+u_{1 \rightarrow 0}\right)$ is vanishing on $\partial V_{0}$. But we also have

$$
\Delta w=-\mu_{0}+\mu_{0}-\mu_{1}+\mu_{1}=0 \text { in } \stackrel{\circ}{V_{0}},
$$

and by the uniqueness of solutions to the Dirichlet problem, we infer that $w=0$ in $V_{0}$. In particular it follows that

$$
\mu_{1}+\Delta u_{1 \rightarrow 0}=\mu_{0}+\Delta u_{0},
$$

which, by the stability of $u_{0}$, implies that $\left(V_{0}, u_{1 \rightarrow 0}\right)$ is stabilizing for $\operatorname{BS}\left(\mu_{1}, \kappa_{0}\right)$. Again, by the minimality principle we get $V_{2} \subset V_{0}$, consequently $V_{2}=V_{0}$. It is left to show (4.4). Since $V_{2}=V_{0}$, the function $u=u_{2}+u_{0 \rightarrow 1}-u_{0}$ vanishes on $\partial V_{0}$ and is identically 0 outside $V_{0}$. Hence in the interior of $V_{0}$ we have

$$
\Delta u=-\mu_{1}+\Delta u_{0 \rightarrow 1}-\Delta u_{0}=-\mu_{1}+\left(\mu_{1}-\mu_{0}\right)+\mu_{0}=0,
$$

which shows that $u=0$ on $\mathbb{Z}^{d}$ and hence (4.4). The lemma is proved.

4.2. Directional monotonicity for point masses. Yet another important consequence of the minimality principle of Theorem 4.2 is a special directional monotonicity of the odometer function corresponding to an initial distribution concentrated at a single point. This key property of the boundary sandpile will prove crucial in studying the regularity of the boundary of scaling limit of the process.

Let $\mathcal{S}$ be the set of mirror symmetry hyperplanes of the unit cube $[0,1]^{d}$. More precisely, $\mathcal{S}$ consists of the hyperplanes $\left\{x_{i}=1 / 2\right\},\left\{x_{i}=x_{j}\right\}$ and $\left\{x_{i}=-x_{j}\right\}$ where $i, j=1, \ldots, d$ with $i \neq j$. In particular, $\mathcal{S}$ has $d^{2}$ elements in total, and the set of vectors (2.9) represents the set of all directions of unit normals to elements of $\mathcal{S}$.

Theorem 4.5. (Directional monotonicity) Assume $(V, u)$ is the stabilizing pair of $\operatorname{BS}\left(n \delta_{0}, \kappa_{0}\right)$ where $n>0$ and $\kappa_{0}>0$. Fix any hyperplane $T \in \mathcal{S}$. Then, for any $X_{1}, X_{2} \in \mathbb{Z}^{d}$, such that $X_{1}-X_{2}$ is a non-zero vector orthogonal to $T$, we have

$$
u\left(X_{1}\right) \geq u\left(X_{2}\right) \quad \text { if } \quad\left|X_{1}\right| \leq\left|X_{2}\right| \text {. }
$$


This result formalizes the intuition that moving away from the origin, where the total mass of the sandpile was concentrated initially, emissions of mass should become smaller. There are however restrictions, partially due to our proof, on the way one moves on the lattice. Namely the theorem allows to compare points lying on a "lattice-line". The proof is motivated by A.D. Alexandrov's celebrated moving plane method [2], see also [29].

Proof of Theorem 4.5. We will assume $\left|X_{1}\right| \leq\left|X_{2}\right|$ and will show that $u\left(X_{1}\right) \geq$ $u\left(X_{2}\right)$. This clearly implies the claim of the theorem.

There is a unique translated copy of $T$, call it $T_{0}$, having equal distance from $X_{1}$ and $X_{2}$. For a given $x \in \mathbb{R}^{d}$ denote by $x^{*}$ its mirror reflection with respect to the hyperplane $T_{0}$. Due to the choice of $T$ and $X_{1}, X_{2}$ it follows that each lattice point is being reflected in this way to a lattice point, and since reflection is an involution, we have that $\left(\mathbb{Z}^{d}\right)^{*}=\mathbb{Z}^{d}$, in particular $X_{1}=\left(X_{2}\right)^{*}$.

Set $V^{*}:=\left\{x^{*}: x \in V\right\}$ for the reflection of $V$, and similarly define the reflected odometer function by $u^{*}(x):=u\left(x^{*}\right)$, where $x \in \mathbb{Z}^{d}$. From the discussion above, we have that $u^{*}$ is defined on $\mathbb{Z}^{d}$ and $V^{*} \subset \mathbb{Z}^{d}$. Let $\mathcal{H}_{-}$be the closed halfspace of $\mathbb{R}^{d}$ determined by $T_{0}$ and containing the origin, and let $\mathcal{H}_{+}=\mathbb{R}^{d} \backslash \mathcal{H}_{-}$. Consider the set $V_{T}=V_{-} \cup V_{+}$where $V_{-}:=\mathcal{H}_{-} \cap V$ and $V_{+}:=\mathcal{H}_{+} \cap V \cap V^{*}$. Our first goal is to show that $V_{T}=V$. By definition $V_{T} \subset V$, so we need to establish the reverse inclusion. Consider the function

$$
u_{T}(x)= \begin{cases}u(x), & \text { if } x \in V_{-}, \\ \min \left\{u(x), u^{*}(x)\right\}, & \text { if } x \in V_{+} .\end{cases}
$$

We claim that

$$
\Delta u_{T}(x) \leq \Delta u(x), \quad x \in \stackrel{\circ}{V}_{T}
$$

Indeed, fix $x$ in the interior of $V_{T}$. There are two possibilities.

Case 1. $x \in V_{-}$.

In this case (4.5) follows from

$$
\begin{aligned}
\Delta u_{T}(x)=\frac{1}{2 d} \sum_{y \sim x, y \in V_{-}} u_{T}(y)+\frac{1}{2 d} \sum_{y \sim x, y \in V_{+}} u_{T}(y)-u(x) \leq \\
\frac{1}{2 d} \sum_{y \sim x, y \in V_{-}} u(y)+\frac{1}{2 d} \sum_{y \sim x, y \in V_{+}} u(y)-u(x)=\Delta u(x) .
\end{aligned}
$$

Case 2. $x \in V_{+}$.

We have two sub-cases here, namely either $u_{T}(x)=u(x)$ or $u_{T}(x)=u^{*}(x)$. In the former case, (4.5) follows as in Case 1 above. We will therefore assume that

$$
u_{T}(x)=\min \left\{u(x), u^{*}(x)\right\}=u^{*}(x) .
$$

Then we have

$$
\begin{aligned}
2 d \Delta u_{T}(x)= & \sum_{y \sim x, y \in V_{-}} u_{T}(y)+\sum_{y \sim x, y \in V_{+}} u_{T}(y)-2 d u^{*}(x) \leq \\
& \sum_{y \sim x, y \in V_{-}} u(y)+\sum_{y \sim x, y \in V_{+}} u^{*}(y)-2 d u^{*}(x)= \\
& \sum_{y \sim x, y \in V_{-}} u(y)+\sum_{y^{*} \sim x^{*}, y \in V_{+}} u\left(y^{*}\right)-2 d u\left(x^{*}\right) .
\end{aligned}
$$


Observe, that if the first sum on the last row of (4.7) is empty, then we get $\Delta u_{T}(x) \leq$ $\Delta u\left(x^{*}\right)$. Otherwise, there are two possible scenarios treated below.

The first one occurs when the hyperplane $T$ is orthogonal to a coordinate axis, i.e. $T$ is one of $\left\{x \in \mathbb{R}^{d}: x_{i}=1 / 2\right\}$ for some $1 \leq i \leq d$. By assumption there is $y \in V_{-}$such that $y \sim x \in V_{+}$. But due to the choice of $T$ this is possible if and only if $y=x^{*}$ and $x^{*} \sim x$. In particular, the above-mentioned sum contains a single element, namely the one arising from $y=x^{*}$. Taking into account (4.6), from (4.7) we obtain $\Delta u_{T}(x) \leq \Delta u\left(x^{*}\right)$.

Consider the second scenario, when the symmetry hyperplane $T$ is not orthogonal to any of the coordinate axes, and assume that there exists $y \in V_{-}$such that $y \sim x \in V_{+}$. Here as well, in view of the choice of $T$, such $y$ must lie on the hyperplane $T_{0}$, and hence $y \sim x^{*}$. Thus the first sum in the last row of (4.7) covers all lattice neighbours of $x^{*}$ lying on $T_{0}$. Observe that all lattice neighbours of $x^{*}$ are in $V_{-}$, so they are either on $T_{0}$, or are obtained as reflections of lattice neighbours of $x$ from $V_{+}$. The first class of neighbours is covered by the first sum, and the second class by the second sum of of the last row of (4.7). Hence, the last row of (4.7) equals $2 d \Delta u\left(x^{*}\right)$.

We conclude that in Case 2

$$
\Delta u_{T}(x) \leq \Delta u\left(x^{*}\right) .
$$

Finally, notice that for $x \in V_{+}$lying in the interior of $V_{T}$ we have that $x^{*}$ is from the interior of $V_{T}$. Hence, $\Delta u\left(x^{*}\right) \in\{0,-n\}$ since the origin is in $V_{-}$. We thus get (4.5) from (4.8). Next, we proceed to the final stage of the proof, where we show that $u \leq u_{T}$ everywhere on $\mathbb{Z}^{d}$.

It is easy to observe that $u_{T}=0$ on $\partial V_{T}$ and $\Delta u_{T} \leq \kappa_{0}$ on $\partial V_{T}$. Now let $v$ be the solution to $\Delta v=-n \delta_{0}$ in the interior of $V_{T}$ and $v=0$ on $\partial V_{T}$. By DMP we get $v \leq u_{T}$ everywhere on $V_{T}$, hence $\Delta v \leq \Delta u_{T} \leq \kappa_{0}$ on $\partial V_{T}$ as both $v$ and $u_{T}$ vanish on the boundary of $V_{T}$. It follows that the pair $\left(V_{T}, v\right)$ is stabilizing for $\operatorname{BS}\left(n \delta_{0}, \kappa_{0}\right)$ (notice that the origin lies in $V_{T}$ ), hence the minimality principle due to Theorem 4.2 in conjunction with the inclusion $V_{T} \subset V$ implies $V_{T}=V$. In particular we get $u \leq u_{T}$ everywhere on $V$ since the difference $u_{T}-u$ defines a supersolution in $V$ with zero boundary data. Lastly, since both $u$ and $u_{T}$ vanish outside $V$, we get that $u \leq u_{T}$ on $\mathbb{Z}^{d}$. From here, the desired inequality follows easily. Indeed, since $\left|X_{1}\right| \leq\left|X_{2}\right|$ and the origin lies in $V_{-}$, we must have $X_{2} \in V_{+}$. Due to construction we also have $X_{1}=\left(X_{2}\right)^{*}$, hence

$$
u\left(X_{2}\right) \leq u_{T}\left(X_{2}\right)=\min \left\{u\left(X_{1}\right), u\left(X_{2}\right)\right\} \leq u\left(X_{1}\right) .
$$

The proof of the theorem is completed.

4.3. Applications to classical Abelian sandpile. The main aim of this subsection is the analysis of the well-known Abelian sandpile model (ASM) via tools developed in this paper. In particular, we will show that the scaling limit of ASM generated from an initial configuration of chips placed at a single vertex has Lipschitz boundary. While some aspects of the local (inner) geometry of ASM were analysed in [23], we believe that this is the first occurrence addressing the geometry of the boundary.

We briefly recall the definition of the process. ASM is a lattice growth model for configurations of chips distributed on vertices of $\mathbb{Z}^{d}$. A vertex carrying at least $2 d$ chips topples giving a single chip to all its $2 d$ lattice neighbours, and losing $2 d$ chips itself. If there are no sites with more than $2 d-1$ chips, the process terminates. 
For any finite non-negative initial configuration of chips, subsequently toppling all sites with at least $2 d$ chips, the process terminates in finite steps. This process is abelian in the sense that the final configuration of chips is independent of the order of topplings.

Fix $n \in \mathbb{N}$, and let $u$ be the odometer function for the starting configuration of chips $n \delta_{0}$. That is, for each $x \in \mathbb{Z}^{d}$ the value $u(x)$ is the number of topplings required to reach stable configuration in ASM. Due to abelian property the function $u_{n}$ is well-defined. Let also $s: \mathbb{Z}^{d} \rightarrow \mathbb{Z}_{+}$be the final (stable) configuration of chips. Similarly to (1.6) one can easily see that

$$
s(x)=n \delta_{0}+\sum_{y \sim x}(u(y)-u(x))=n \delta_{0}+2 d \Delta u(x), \quad x \in \mathbb{Z}^{d} .
$$

We will need the following characterization proved by Fey, Levine, and Peres [9].

Least Action Principle. Let $n$ and $u$ be as above. Then

$$
u=\min \left\{w: \mathbb{Z}^{d} \rightarrow \mathbb{Z}_{+}: n \delta_{0}+2 d \Delta w \leq 2 d-1\right\} .
$$

We have the analogue of Theorem 4.5 for ASM. For the definition of $\mathcal{S}$ see the paragraph above Theorem 4.5.

Theorem 4.6. (Directional monotonicity for ASM) For a given $n \in \mathbb{N}$ let $u$ be the odometer for initial distribution $n \delta_{0}$. Fix any hyperplane $T \in \mathcal{S}$. Then, for any $X_{1}, X_{2} \in \mathbb{Z}^{d}$, such that $X_{1}-X_{2}$ is a non-zero vector orthogonal to $T$, we have

$$
u\left(X_{1}\right) \geq u\left(X_{2}\right) \quad \text { if } \quad\left|X_{1}\right| \leq\left|X_{2}\right| \text {. }
$$

Proof of Theorem 4.6. We will only sketch the proof as it is a simplification of the proof of Theorem 4.5. Assuming $\left|X_{1}\right| \leq\left|X_{2}\right|$ will show that $u\left(X_{1}\right) \geq u\left(X_{2}\right)$, which implies the claim of the theorem.

Let $T_{0}$ be as in the proof of Theorem 4.5, and for a given $x \in \mathbb{R}^{d}$ denote by $x^{*}$ its mirror reflection with respect to the hyperplane $T_{0}$. Recall that we have $X_{1}=\left(X_{2}\right)^{*}$. Define also the reflected odometer function by $u^{*}(x):=u\left(x^{*}\right)$, where $x \in \mathbb{Z}^{d}$. We have that $u^{*}$ is defined on $\mathbb{Z}^{d}$ with values in $\mathbb{Z}_{+}$. Let $\mathcal{H}_{-}$be the closed halfspace of $\mathbb{R}^{d}$ determined by $T_{0}$ and containing the origin, and let $\mathcal{H}_{+}=\mathbb{R}^{d} \backslash \mathcal{H}_{-}$. Set $V_{-}:=\mathcal{H}_{-} \cap \mathbb{Z}^{d}, V_{+}:=\mathcal{H}_{+} \cap \mathbb{Z}^{d}$, and define the function

$$
u_{T}(x)= \begin{cases}u(x), & \text { if } x \in V_{-}, \\ \min \left\{u(x), u^{*}(x)\right\}, & \text { if } x \in V_{+} .\end{cases}
$$

We aim at showing that

$$
n \delta_{0}+2 d \Delta u_{T}(x) \leq 2 d-1, \quad x \in \mathbb{Z}^{d} .
$$

First observe, that this inequality clearly implies the result of the theorem. Indeed, from (4.9) and the least action principle formulated above we obtain $u \leq u_{T}$ on $\mathbb{Z}^{d}$. Next, since $\left|X_{1}\right| \leq\left|X_{2}\right|$ and the origin lies in $V_{-}$, we must have $X_{2} \in V_{+}$. Due to construction we also have $X_{1}=\left(X_{2}\right)^{*}$, hence

$$
u\left(X_{2}\right) \leq u_{T}\left(X_{2}\right)=\min \left\{u\left(X_{1}\right), u\left(X_{2}\right)\right\} \leq u\left(X_{1}\right),
$$

and the result follows. It is thus left to establish (4.9), which we do next. Fix any $x \in \mathbb{Z}^{d}$. There are two possibilities.

Case 1. $x \in V_{-}$. Similarly to Case 1 of Theorem 4.5 we get

$$
2 d \Delta u_{T}(x) \leq 2 d \Delta u(x) \leq 2 d-1-n \delta_{0},
$$

where the last inequality is due to definition of $u$. 
Case 2. $x \in V_{+}$. Exactly, as in Case 2 of Theorem 4.5 we get

$$
2 d \Delta u_{T}(x) \leq 2 d \Delta u\left(x^{*}\right) \leq 2 d-1 .
$$

The proof of the theorem is completed.

For each integer $n \in \mathbb{N}$ let $u_{n}$ and $s_{n}$ be respectively the odometer, and final configuration of chips for initial distribution $n \delta_{0}$. Define $\bar{u}_{n}(x)=n^{-2 / d} u_{n}\left(n^{1 / d} x\right)$ and $\bar{s}_{n}(x)=s_{n}\left(n^{1 / d} x\right)$ where $x \in n^{-1 / d} \mathbb{Z}^{d}$. It was proved by Pegden and Smart [28] that there exists a non-negative and compactly supported function $u_{0} \in C\left(\mathbb{R}^{d} \backslash\{0\}\right)$, $s_{0} \in L^{\infty}\left(\mathbb{R}^{d}\right)$ satisfying $0 \leq s_{0} \leq 2 d-1$ such that $\bar{u}_{n} \rightarrow u_{0}$ locally uniformly in $\mathbb{R}^{d} \backslash\{0\}, \bar{s}_{n} \rightarrow s_{0}$ weak $^{*}$ in $L^{\infty}\left(\mathbb{R}^{d}\right)$ and $\Delta u_{0}=s_{0}-\delta_{0}$ in the sense of distributions, where $\Delta$ is continuous Laplacian and $\delta_{0}$ is Dirac delta at the origin. The reader is referred to [28] for the details.

Concerning ASM, we prove the following statement.

Theorem 4.7. For $u_{0}$ as above set $u_{0}(0)=+\infty$ and let the set of directions $\mathcal{N}$ be defined as in (2.9). The following is true:

(a) for any $X_{1}, X_{2} \in \mathbb{R}^{d}$ with the property that $X_{2}-X_{1}$ is non-zero and is collinear to any of the vectors of $\mathcal{N}$, we have

$$
u_{0}\left(X_{1}\right) \geq u_{0}\left(X_{2}\right) \quad \text { if } \quad\left|X_{1}\right| \leq\left|X_{2}\right| .
$$

(b) $\partial\left\{u_{0}>0\right\}$ is a locally Lipschitz graph.

The reason for defining $u_{0}(0)=+\infty$ is mainly for the mathematical rigour in the proof. Apart from monotonicity of the limiting odometer function, this result shows that the boundary of the scaling limit is Lipschitz. We postpone the proof of this theorem until subsection 5.2.

Remark 4.8. This method seems to be promising also for Abelian Sandile model with background heights [9].

4.4. The true scale of the model. The main aim of this section is to study the growth of the boundary sandpile process for point masses.

4.4.1. Heuristics. It seems intuitively clear that the value $\kappa_{0}$ of boundary capacity should influence the size of the visited sites. Specifically, the smaller $\kappa_{0}$ the greater number of boundary points will be necessary in the stable configuration, resulting in a larger domain of visited sites. Our aim is to determine, for initial distributions concentrated at the origin, the size of the boundary capacity which will leave a chance for existence of a scaling limit. To proceed, let $\left(V_{n}, u_{n}\right)$ be the stabilizing pair for $\operatorname{BS}\left(n \delta_{0}, \kappa_{0}\right)$, with $n>1$ large. Let $\sigma_{n}$ be the mass distribution in the stable configuration. By definition we have that $\sigma_{n}$ is concentrated on $\partial V_{n}$, and the odometer function $u_{n}$ satisfies

$$
\Delta u_{n}(x)=\sigma_{n}(x)-n \delta_{0}, \quad x \in \mathbb{Z}^{d} .
$$

After scaling the lattice by a small factor $h=h(n)>0$ we will get

$$
\Delta^{h} \widetilde{u}_{n}(x)=\sigma_{n}\left(h^{-1} x\right)-n \delta_{0}, \quad x \in h \mathbb{Z}^{d},
$$

where $\widetilde{u}_{n}(x):=h^{2} u_{n}\left(h^{-1} x\right)$ is the scaled odometer defined on $h \mathbb{Z}^{d}$. The scaling limit amount to taking $n \rightarrow \infty$. We then want $n \delta_{0}$ to converge in the sense of distributions to a continuous Dirac at the origin. Since the volume element at the scale $h>0$ is $h^{d} d x$ we need $n h^{d}=1$ hence the correct order of scaling would be $h=n^{-1 / d}$. But as $h \rightarrow 0$ we want $h V_{n}$ to neither shrink to a lower dimensional 
object, nor escape to infinity, hence $V_{n}$ should be of uniform size ${ }^{12} h^{-1}$. The size of $V_{n}$ will be determined by $\kappa_{0}$. Indeed, the argument of Lemma 4.12 shows that for given $\kappa_{0}$, and $n>1$ large, $V_{n}$ would have size $R$ where $\kappa_{0} \approx n R^{1-d}$. But for the size $R$ we require $R \approx h=n^{1 / d}$, therefore $\kappa_{0} \approx n^{1 / d}$ is the only choice for boundary capacity to facilitate this requirement.

In the light of these arguments, in dimension $d \geq 2$ we will set $\kappa_{0}=n^{1 / d}$ for initial mass $n>1$ at the origin.

Lemma 4.9. (Monotone and unbounded growth) For $n \geq 1$ let $V_{n}$ be the set of visited sites of $\mathrm{BS}\left(n \delta_{0}, n^{1 / d}\right)$. We have the following assertions:

(a) if $1 \leq n<m$ then $V_{n} \subset V_{m}$.

(b) $\bigcup_{n \geq 1} V_{n}=\mathbb{Z}^{d}$.

Proof. We start with (a). Let $\partial V_{m}=\left\{x^{(i)}\right\}_{i=1}^{k}$ for some $k \in \mathbb{N}$. Since the Laplacian is a linear operator (cf. (3.5)), there is a collection of weights $\left\{\alpha_{i}\right\}_{i=1}^{k}$ with all $\alpha_{i}>0$ such that for any mass $N>0$ at the origin and $\partial V_{m}$ as target set, each site $x^{(i)}$ receives mass $\alpha_{i} N$.

By definition we have that $V_{m}$ is stable, hence $\alpha_{i} m \leq \kappa_{0}(m)=m^{1 / d}$ and we get $\alpha_{i} \leq m^{1 / d-1}$ for all $1 \leq i \leq k$. From here it follows

$$
\alpha_{i} n \leq m^{1 / d-1} n \leq n^{1 / d-1} n=n^{1 / d}=\kappa_{0}(n),
$$

which means that $V_{m}$ is stable for mass $n$ at the origin. But as $V_{n}$ is the smallest among all stable domains for mass $n$ in view of Theorem 4.2, we get $V_{n} \subset V_{m}$, completing the proof of part (a).

We proceed to part (b). Let $n \geq 1$ be fixed, then in view of (a) we have $V_{n} \subset V_{m}$ for any $m>n$. Hence from Lemma 4.4 to produce $V_{m}$ we may topple mass $m$ from the origin to $\partial V_{n}$ and then stabilize $\partial V_{n}$ in $V_{m}$. As in part (a) we know that each $x^{(i)} \in \partial V_{n}$ gets mass $\alpha_{i} m$. Therefore, for $m>n$ large enough, we will get $\alpha_{i} m>m^{1 / d}=\kappa_{0}(m)$ for all $1 \leq i \leq k$. As a result, all points on $\partial V_{n}$ have to topple in order to stabilize $V_{m}$. This proves (b), and finishes the proof of the lemma.

Lemma 4.10. For $n \geq 1$ let $(V, u)$ be the stabilizing pair of $\operatorname{BS}\left(n \delta_{0}, \kappa_{0}\right)$. If $B$ is a subset of $V$ containing the origin in its interior and $v: B \rightarrow \mathbb{R}$ is such that

$$
\begin{cases}\Delta v \geq-n \delta_{0}, & \text { in } \stackrel{\circ}{B} \\ v=0, & \text { on } \partial B \\ \Delta v>\kappa_{0}, & \text { on } \partial B\end{cases}
$$

then $\partial B \cap \partial V=\emptyset$.

Proof. Assume for contradiction that there exists $x_{0} \in \partial V \cap \partial B$, and consider the function $w=u-v$ with $u, v$ as above. Then clearly $\Delta w \leq 0$ in $\stackrel{\circ}{B}$ and $w \geq 0$ on $\partial B$, hence by DMP we get $w \geq 0$ in $B$, i.e. $u \geq v$ in $B$. Since both $u$ and $v$ vanish at $x_{0}$ we obtain

$$
\kappa_{0}<\Delta v\left(x_{0}\right)=\frac{1}{2 d} \sum_{y \sim x_{0}} v(y) \leq \frac{1}{2 d} \sum_{y \sim x_{0}} u(y)=\Delta u\left(x_{0}\right) \leq \kappa_{0},
$$

\footnotetext{
${ }^{12}$ By size $h^{-1}$ we mean that $B\left(0, c_{1} h^{-1}\right) \cap \mathbb{Z}^{d} \subset V_{n} \subset B\left(0, c_{2} h^{-1}\right)$ where $B(0, r)$ is the Euclidean ball centred at the origin, and having radius $r>0$, and $0<c_{1}<c_{2}$ are absolute constants.
} 
where the last inequality follows by stability of the sandpile. This leads to a contradiction and completes the proof.

For $R>0$ let $G_{R}$ be the Green's function with pole at the origin for the discrete ball $Z_{R}$ defined in (1.4), i.e.

$$
\Delta G_{R}=-\delta_{0} \text { in } \stackrel{\circ}{Z}_{R} \quad \text { and } \quad G_{R}=0 \text { on } \partial Z_{R} .
$$

Lemma 4.11. There exist constants $R_{0}>0$ depending on dimension only, and $0<c_{1}<C_{1}$ depending on $R_{0}$ and dimension $d$, such that for any $R>R_{0}$ one has

$$
c_{1} R^{1-d}<\Delta G_{R}(x)<C_{1} R^{1-d} \quad \forall x \in \partial Z_{R} .
$$

Proof. The proof is a straightforward consequence of the random walk counterpart of the problem. Let $g(x, y): \mathbb{Z}^{d} \times \mathbb{Z}^{d} \rightarrow \mathbb{R}$ be the fundamental solution to $\Delta$ in $\mathbb{Z}^{d}$, i.e. $\Delta_{x} g(x, y)=-\delta_{0}(y-x)$ for all $x, y \in \mathbb{Z}^{d}$. The following asymptotics is well-known [13], [30], [21]

$$
g(x, y)= \begin{cases}-\frac{2}{\pi} \log |x-y|+\gamma_{0}+O\left(|x-y|^{-2}\right), & d=2, \\ \frac{2}{(d-2) \omega_{d}}|x-y|^{2-d}+O\left(|x-y|^{-d}\right), & d \geq 3,\end{cases}
$$

where $\gamma_{0}$ is a constant, and $\omega_{d}$ is the volume of the unit ball in $\mathbb{R}^{d}$. By $[22$, Proposition 4.6.2] for each $x \in \stackrel{\circ}{Z}_{R}$ we have

$$
G_{R}(x)=g(x, 0)-\mathbb{E}^{x}\left[g\left(S_{\tau_{R}}, 0\right)\right],
$$

where $\tau_{R}$ is the first exit time from $\stackrel{\circ}{Z}_{R}$ of a simple random walk $\left\{S_{k}\right\}_{k=0}^{\infty}$ on $\mathbb{Z}^{d}$ started at $x \in \stackrel{\circ}{Z}_{R}$, i.e. $\tau_{R}=\min \left\{k \geq 1: S_{k} \notin \stackrel{\circ}{Z}_{R}\right\}$. Expanding the expectation in (4.12) and using the fact that the simple random walk started inside a finite set of $\mathbb{Z}^{d}$ will almost surely exit it in finite time, we get

$$
G_{R}(x)=\sum_{z \in \partial Z_{R}} \mathbb{P}^{x}\left[S_{\tau_{R}}=z\right](g(x, 0)-g(z, 0)) .
$$

For $x \in \stackrel{\circ}{Z}_{R}$ satisfying $|x| \geq R / 2$, applying (4.11) to the last formula for $G_{R}$ we obtain

$$
G_{R}(x)=c_{d} \widetilde{G}_{R}(x)+O\left(R^{-d}\right),
$$

where

$$
\widetilde{G}_{R}(x)=\sum_{z \in \partial Z_{R}} \mathbb{P}^{x}\left[S_{\tau_{R}}=z\right] \begin{cases}\log \frac{|z|}{|x|}, & d=2, \\ \frac{1}{|x|^{d-2}}-\frac{1}{|z|^{d-2}}, & d \geq 3,\end{cases}
$$

and $c_{d}$ is a constant depending on $d$. Now things are reduced to some elementary computations.

We start with a lower bound of the lemma. Consider the case of $d=2$. Fix a point $M_{0}=(a, b) \in \check{Z}_{R}$ such that there exists $M_{1} \sim M_{0}$ with $M_{1} \in \partial Z_{R}$. Let $\max \{|a|,|b|\}=|a|$. In view of the definition of $Z_{R}$, the neighbour of $M_{0}$ having the largest norm among all 4 neighbours must be on the boundary of $Z_{R}$. Since we also have $|a| \geq|b|$, it follows that as $M_{1}$ one can take $(a+1, b)$ for $a>0$ and $(a-1, b)$ 
for $a<0$. Assume we have $M_{1}=(a+1, b)$, i.e. $a>0$. Then

$$
\begin{aligned}
\log \frac{\left|M_{1}\right|}{\left|M_{0}\right|}=\frac{1}{2} \log \frac{\left|M_{1}\right|^{2}}{\left|M_{0}\right|^{2}} & =\frac{1}{2} \log \left(1+\frac{2 a+1}{a^{2}+b^{2}}\right)=\frac{1}{2} \frac{2 a+1}{a^{2}+b^{2}}+O\left(a^{-2}\right) \geq \\
\frac{1}{a}+ & O\left(a^{-2}\right) \geq \frac{1}{\sqrt{2}} \frac{1}{\left|M_{0}\right|}+O\left(\left|M_{0}\right|^{-2}\right) \geq \frac{1}{\sqrt{2}} R^{-1}+O\left(R^{-2}\right) .
\end{aligned}
$$

Observe that $\mathbb{P}^{M_{0}}\left\{S_{\tau_{R}}=M_{1}\right\} \geq 1 / 4$ since the random walk can exit from $\stackrel{\circ}{R}_{R}$ in one step through $M_{1}$ if started at $M_{0}$. On the other hand, from the definition of $Z_{R}$ we have $|x|<|z|$ for any $x \in \check{Z}_{R}$ and any $z \in \partial Z_{R}$, hence all terms in the sum of $\widetilde{G}_{R}$ are non-negative. Using this, for $M_{0}$ and $M_{1}$ as above we get

$$
\widetilde{G}_{R}\left(M_{0}\right) \geq \frac{1}{4} \log \frac{\left|M_{1}\right|}{\left|M_{0}\right|}+O\left(R^{-2}\right) \geq \frac{1}{4 \sqrt{2}} R^{-1}+O\left(R^{-2}\right) .
$$

This implies $G_{R}\left(M_{0}\right) \geq c_{2} R^{-1}$ for any $M_{0} \in \stackrel{\circ}{Z}_{R}$ having a neighbour on $\partial Z_{R}$. Since $G_{R}$ vanishes on $\partial Z_{R}$ the bound of the lemma follows.

The lower bound in case of $d \geq 3$ is handled similarly. The upper bound of the lemma follows directly from (4.13)-(4.14) and definition of the ball $Z_{R}$. The details, which we will omit, are elementary. The proof of the lemma is complete.

Lemma 4.12. There are dimension dependent constants $0<c_{1}<C_{1}$ such that if $V_{n}$ is the set of visited sites of $\operatorname{BS}\left(n \delta_{0}, n^{1 / d}\right)$, with $n>1$ large, then

$$
Z_{c_{1} n^{1 / d}} \subset V_{n} \subset Z_{C_{1} n^{1 / d}} .
$$

Proof. By Lemma 4.9 part (b) every site of $\mathbb{Z}^{d}$ is eventually visited by the sandpile if we keep increasing $n$. Thus we will assume that $n>1$ is so large that the discrete ball $Z_{2 R_{0}}$ is inside $V_{n}$, where $R_{0}$ is fixed from Lemma 4.11. Let us also remark, that if for some $n>1$ we have the inclusion $Z_{2 R_{0}} \subset V_{n}$, then for all $n^{\prime}>n$ we get $Z_{2 R_{0}} \subset V_{n^{\prime}}$ in view of Lemma 4.9 part (a).

Let $G_{R}$ be as above (see (4.10)). Then, the function $G_{R, n}:=n G_{R}$ satisfies

$$
\Delta G_{R, n}=-n \delta_{0} \text { in } \stackrel{\circ}{Z}_{R} \text { and } G_{n, R}=0 \text { on } \partial Z_{R} .
$$

From Lemma 4.11 we have $\Delta G_{R, n} \geq c_{1} n R^{1-d}$ everywhere on $\partial Z_{R}$. Observe that if $Z_{R} \subset V_{n}$ and $n$ and $R$ are chosen so that $\Delta G_{R, n}>n^{1 / d}$ on $\partial Z_{R}$, then by Lemma 4.10 we have $\partial Z_{R} \cap \partial V_{n}=\emptyset$, i.e. $Z_{R}$ stays strictly inside $V_{n}$. We now choose a sequence of radii $R_{0}<R_{1}<\ldots<R_{k}$, where $R_{0}$ is as above, and for each $1 \leq i \leq k$ we have $Z_{R_{i}} \subset Z_{R_{i+1}}$ and $Z_{R_{i+1}} \backslash Z_{R_{i}} \subset \partial Z_{R_{i+1}}$, where the latter simply means that we enlarge the balls by 1 lattice step at most. Given this, as long as we have $c_{1} n R_{i}^{1-d}>n^{1 / d}$ we get $Z_{R_{i}} \subset V_{n}$. We can therefore increase the radius up to $c_{1}^{1 /(d-1)} n^{1 / d}$ obtaining by so the first inclusion of the current lemma.

The upper bound can be obtained similarly, by starting with a ball of sufficiently large radius, containing $V_{n}$, then shrinking the ball by at most 1 lattice step at a time and using the upper bound of Lemma 4.11 instead. The proof of the lemma is complete.

Our next result gives a uniform lower bound on the odometer function, which will be needed in the analysis of the shape of scaling limit. For the proof we need a discrete version of Harnack's inequality. 
Theorem 4.13. (Harnack inequality, see [21, Theorem 1.7.2]) For any $\alpha<1$ there exists a constant $C_{\alpha}$ such that if $f: Z_{R} \rightarrow[0, \infty)$ is harmonic in $\stackrel{\circ}{Z}_{R}$ then $f\left(x_{1}\right) \leq$ $C_{\alpha} f\left(x_{2}\right)$ for any $\left|x_{1}\right|,\left|x_{2}\right| \leq \alpha R$.

Lemma 4.14. (Bounds from below) For any $r_{0}>0$ small there exists a constant $c_{0}>0$ depending on $r_{0}$ and dimension $d$ such that for any $n>1$ and each $x^{(0)} \in V_{n}$ satisfying $\operatorname{dist}\left(x^{(0)}, \partial V_{n}\right) \geq r_{0} n^{1 / d}$ one has

$$
u_{n}\left(x^{(0)}\right) \geq c_{0} n^{2 / d}
$$

where $\left(V_{n}, u_{n}\right)$ is the stabilizing pair of $\operatorname{BS}\left(n \delta_{0}, n^{1 / d}\right)$.

Proof. We first observe that the bound of the lemma holds near the origin, i.e. there exist dimension dependent constants $\alpha_{0}>0$ and $c_{0}$ such that for any $n>1$ one has

$$
u_{n}(x) \geq c_{0} n^{2 / d}, \quad x \in Z_{\alpha_{0} n^{1 / d}} .
$$

Indeed, by Lemma 4.12 and DMP we have $u_{n}(x) \geq n G_{c_{1} n^{1 / d}}(x)$ for some constant $c_{1}=c_{1}(d)$, where the Green's function $G$ is defined in (4.10). Thanks to this bound, (4.15) follows directly from asymptotics of Green's function proved in [21, Proposition 1.5.9] for $d \geq 3$, and [21, Proposition 1.6.7] for $d=2$. Next, we reduce the general case of the lemma to (4.15) by constructing a Harnack chain leading from a given point to the ball $Z_{r_{0} n^{1 / d}}$.

Let $x^{(0)}=\left(a_{1}, a_{2}, \ldots, a_{d}\right) \in \mathbb{Z}^{d}$ and assume $x^{(0)} \notin Z_{\alpha_{0} n^{1 / d}}$, since otherwise the estimate follows by (4.15). Suppose $\left|a_{d}\right|=\max _{1 \leq i \leq d}\left|a_{i}\right|$, and denote $\beta_{0}:=$ $\min \left\{\alpha_{0} / 4, r_{0} / 4\right\}$, where $\alpha_{0}$ is fixed from (4.15) and $r_{0}$ from the formulation of the lemma. We get that the ball

$$
Z_{0}:=\left\{x \in \mathbb{Z}^{d}:\left|x-x^{(0)}\right| \leq \beta_{0} n^{1 / d}\right\}
$$

lies inside $V_{n} \backslash\{0\}$. By $\Pi_{d}$ denote the orthogonal projection of $Z_{0}$ onto $\mathbb{Z}^{d-1} \times\{0\}$, i.e.

$$
\Pi_{d}:=\left\{\left(x_{1}, \ldots, x_{d-1}, 0\right) \in \mathbb{Z}^{d}: \exists a \in \mathbb{Z} \text { s.t. }\left(x_{1}, \ldots, x_{d-1}, a\right) \in Z_{0}\right\} .
$$

By directional monotonicity of Theorem 4.5 applied to the direction $e_{d}$, and axial symmetry of the odometer given by Corollary 2.4, we have

$$
\mathcal{C}_{d}:=\left(\Pi_{d} \times\left[-a_{d}, a_{d}\right]\right) \cap \mathbb{Z}^{d} \subset V_{n} .
$$

If the cylinder $\mathcal{C}_{d}$ intersects the ball $Z_{\alpha_{0} n^{1 / d}}$, the estimate of the lemma follows by Harnack's inequality of Theorem 4.13 and upper estimate of Lemma 4.12, where the latter is used to estimate the length of Harnack chain. Otherwise, if $\mathcal{C}_{d} \cap Z_{\alpha_{0} n^{1 / d}}=\emptyset$, denote $x^{(1)}=\left(a_{1}, \ldots, a_{d-1}, 0\right)$, and $Z_{1}=\left\{x \in \mathbb{Z}^{d}:\left|x-x^{(1)}\right| \leq \beta_{0} n^{1 / d}\right\}$. By construction $Z_{1} \subset \mathcal{C}_{d} \subset V_{n}$. We have, due to Harnack's inequality, that $u_{n}\left(x^{(1)}\right) \geq$ $C u_{n}\left(x^{(0)}\right)$ with a constant $C=C\left(d, r_{0}\right)$, hence it is enough to prove the lemma for $x^{(0)}$ replaced by $x^{(1)}$. For that, we apply the same argument as we had for $x^{(0)}$ to $x^{(1)}$. Since $x^{(1)}$ has at most $d-1$ non-zero coordinates, this reduction procedure on coordinates will terminate in at most $d-1$ number of steps, where in the last step, the corresponding cylinder (4.16) will intersect the ball $Z_{\alpha_{0} n^{1 / d}}$ implying the desired estimate of the lemma. The proof is complete. 


\section{Shape ANALYSis}

The purpose of this section is the study of scaling limit of the model when the initial distribution is concentrated at a point. We will show that after proper scaling of mass and the underlying lattice, there is a convergence along subsequences. The approach is via uniform gradient bounds for the odometers. Combined with some results of the previous section, we will prove a certain non-degeneracy result of the converging shapes, as well as Lipschitz regularity of the boundary of scaling limit.

Throughout this section, we assume that initial distribution of the sandpile equals $n \delta_{0}$, where $n>1$ and boundary capacity of the model is $n^{1 / d}$. For all $n>1$, by $u_{n}$ we denote the corresponding odometer function and by $V_{n}$ the set of visited sites.

5.1. Gradient bounds. First, we establish uniform Lipschitz bounds on the odometer functions away from the origin.

Lemma 5.1. Let $r_{0}>0$ be any fixed number. Then, there exists a constant $C=$ $C\left(r_{0}\right)$ such that for any $n>1$ one has

$$
\left|u_{n}(x)-u_{n}(y)\right| \leq C n^{1 / d}
$$

where $x, y \in V_{n}$ satisfy $r_{0} n^{1 / d} \leq|x| \leq 2 r_{0} n^{1 / d}$ and $x \sim y$.

Proof. Let $G_{n}(x, y)$ be the Green's function for $V_{n}$, i.e. $\Delta_{x} G_{n}(x, y)=-\delta_{0}(y-x)$ for any $x, y \in \stackrel{\circ}{V}_{n}$ and $G(x, y)=0$ if $x \in \partial V_{n}$ or $y \in \partial V_{n}$. From [22, Proposition 4.6.2] for $x, y \in \stackrel{\circ}{V}_{n}$ we have

$$
G_{n}(x, y)=\mathbb{E}^{x}\left[g\left(S_{\tau}, y\right)\right]-g(x, y),
$$

where as before $g$ is defined from (4.11), and $\tau$ is the first exit time from $\stackrel{\circ}{V}_{n}$ of the simple random walk $\left\{S_{k}\right\}_{k=0}^{\infty}$ started at $x$. From the definition of $G_{n}$ we have

$$
\begin{array}{r}
\left|G_{n}(0, x)-G_{n}(0, y)\right| \leq|g(0, x)-g(0, y)|+\mathbb{E}^{x}\left|g\left(S_{\tau}, x\right)-g\left(S_{\tau}, y\right)\right| \leq \\
C\left(|x|^{1-d}+n^{-1}+\max _{w \in \partial V_{n}}|g(w, x)-g(w, y)|\right),
\end{array}
$$

where $C$ is a dimension dependent constant, and we have used the asymptotics (4.11) and Mean-Value Theorem along with the choice of $x$ and $y$ to bound the first summand. From Lemma 4.12 and the choice of $x, y$ we get that $|x-w| \geq c_{d} n^{1 / d}$ for any $w \in \partial V_{n}$. This, coupled with (4.11) and Mean-Value Theorem, for any $w \in \partial V_{n}$ implies

$$
|g(w, x)-g(w, y)| \leq C \frac{|x-y|}{|w-x|^{d-1}}+C n^{-1} \leq C n^{-1+1 / d} .
$$

Combining this estimate with (5.1) we obtain

$$
\left|G_{n}(0, x)-G_{n}(0, y)\right| \leq C n^{-1+1 / d} .
$$

Since $G_{n}(x, y)=G_{n}(y, x)$ we get from the definition of $u_{n}$ that $u_{n}(x)=n G_{n}(0, x)$ for all $x \in V_{n}$, which together with (5.2) completes the proof of the lemma.

Proposition 5.2. (Uniform Lipschitz bound) For any $r_{0}>0$ there exists a constant $C=C\left(r_{0}\right)$ such that for any $n>1$ and all $x, y \in \mathbb{Z}^{d}$ satisfying $|x|,|y|>r_{0} n^{1 / d}$ one has

$$
\left|u_{n}(x)-u_{n}(y)\right| \leq C n^{1 / d}|x-y|
$$


Proof. Recall that $V_{n} \subset \mathbb{Z}^{d}$ is the set of visited sites for mass $n>1$. Define

$$
V_{n, 0}=\left\{x \in V_{n}:|x| \geq r_{0} n^{1 / d} \text { and } \operatorname{dist}\left(x, \partial V_{n}\right) \geq 2\right\},
$$

where dist is the combinatorial distance. For each $1 \leq i \leq d$ consider the discrete derivative of $u$, namely $\partial_{i}^{+} u(x):=u\left(x+e_{i}\right)-u(x)$ with $x \in \mathbb{Z}^{d}$. Clearly $\partial_{i}^{+} u$ is harmonic in $V_{n, 0}$. Now, in view of the stability of the sandpile, for any $x \in V_{n}$ such that $\operatorname{dist}\left(x, \partial V_{n}\right) \leq 1$ we have $\left|\partial_{i}^{+} u(x)\right| \leq C_{1} n^{1 / d}$ with a constant $C_{1}=C_{1}(d)$. On the other hand, by Lemma 5.1 we have $\left|\partial_{i}^{+} u(x)\right| \leq C_{r_{0}} n^{1 / d}$ if $r_{0} n^{1 / d} \leq|x| \leq 2 r_{0} n^{1 / d}$. Since $\Delta\left(\partial_{i}^{+} u\right)=0$ in the interior of $V_{n, 0}$, by DMP we get $\left|\partial_{i}^{+} u(x)\right| \leq C n^{1 / d}$ in $V_{n, 0}$. The same bound obviously works for $\partial_{i}^{-} u(x):=u\left(x-e_{i}\right)-u(x)$. We have thus proved 1-step Lipschitz bound, i.e. the estimate of the proposition if $x$ and $y$ are lattice neighbours.

For the general case, take any $x, y$ such that $|x|,|y|>r_{0} n^{1 / d}$, and consider the shortest lattice path connecting $x$ and $y$ and staying outside the ball $B_{r_{0} n^{1 / d}}$. Namely, let

$$
x=X_{0} \sim X_{1} \sim \ldots \sim X_{k}=y,
$$

where $X_{i} \in \mathbb{Z}^{d}$ and $\left|X_{i}\right|>r_{0} n^{1 / d}$. Clearly such path exists. It is also clear that for the length of the path we have $k \approx|x-y|$ where equivalence is with dimension dependent constants. Using this and the 1-step Lipschitz bound already proved above, we obtain

$$
|u(x)-u(y)| \leq \sum_{i=0}^{k-1}\left|u\left(X_{i+1}\right)-u\left(X_{i}\right)\right| \leq C_{r_{0}} n^{1 / d} k \leq C_{r_{0}} n^{1 / d}|x-y|,
$$

completing the proof of the proposition.

5.2. Scaled odometers. For $n \geq 1$ set $h=n^{-1 / d}$, and define the scaled odometer by $u_{h}(x)=h^{2} u_{n}\left(h^{-1} x\right)$ where $x \in h \mathbb{Z}^{d}$. Let also $V_{h}:=h V_{n} \subset h \mathbb{Z}^{d}$ be the scaled set of visited sites for mass $n$. Clearly, $u_{h}$ is supported in the interior of $V_{h}$. Moreover, in view of Lemma 4.12 we have that the sets $\left\{V_{h}\right\}_{0<h \leq 1}$, are uniformly bounded and contain a ball of some fixed radius.

We will need a few notation. For $0<h \leq 1$ and $\xi=\left(\xi_{1}, \ldots, \xi_{d}\right) \in h \mathbb{Z}^{d}$ define the half-open cube

$$
\mathrm{C}_{h}(\xi)=\left[\xi_{1}-\frac{h}{2}, \xi_{1}+\frac{h}{2}\right) \times \ldots \times\left[\xi_{d}-\frac{h}{2}, \xi_{d}+\frac{h}{2}\right),
$$

and for a given set $V \subset h \mathbb{Z}^{d}$ define $V^{\square}=\bigcup_{\xi \in V} \mathrm{C}_{h}(\xi)$.

In order to study the scaling limit of the model, we need to extend each $u_{h}$ to a function defined on $\mathbb{R}^{d}$. We will use a standard extension of $u_{h}$ which preserves its $\Delta^{h}$-Laplacian. Namely, for fixed $0<h \leq 1$ define a function $u_{h}: \mathbb{R}^{d} \rightarrow \mathbb{R}_{+}$, where for each $\xi \in h \mathbb{Z}^{d}$ and any $x \in \mathrm{C}_{h}(\xi)$ we have set $u_{h}(x)=u_{h}(\xi)$. Clearly $\Delta^{h} u_{h}(x)=\Delta^{h} u_{h}(\xi)$ for all $x \in \mathrm{C}_{h}(\xi)$. Note that we are using the same notation for extended odometers. In what follows $u_{h}$ will stand for this extension. Also, for a given set $E \subset \mathbb{R}^{d}$ we write $\stackrel{\circ}{E}$ for the interior of $E$ in the next theorem. The following is our main result concerning scaling limit of the model.

Theorem 5.3. There exists a sequence $h_{k} \rightarrow 0$, and compactly supported nonnegative function $u_{0} \in C\left(\mathbb{R}^{d} \backslash\{0\}\right)$ which is Lipschitz outside any neighbourhood of the origin, such that 
(i) $u_{h_{k}} \rightarrow u_{0}$ locally uniformly in $\mathbb{R}^{d} \backslash\{0\}$,

(ii) $\Delta u_{0}=-\delta_{0}$ in $\left\{u_{0}>0\right\}$ in the sense of distributions, where $\delta_{0}$ is the Dirac delta at the origin and $\Delta$ denotes the continuous Laplace operator,

(iii) if $V_{0} \subset \mathbb{R}^{d}$ is the support of $u_{0}$ then, $V_{0}=\bigcup_{m=1}^{\infty} \bigcap_{k=m}^{\infty} V_{h_{k}}^{\square}$,

(iv) if we set $u_{0}(0)=+\infty$, and let the set of vectors $\mathcal{N}$ be defined as in (2.9), then for any $x^{(1)}, x^{(2)} \in \mathbb{R}^{d}$ with the property that $x^{(2)}-x^{(1)}$ is non-zero and is collinear to any of the vectors of $\mathcal{N}$, we have

$$
u_{0}\left(x^{(1)}\right) \geq u_{0}\left(x^{(2)}\right) \quad \text { if } \quad\left|x^{(1)}\right| \leq\left|x^{(2)}\right| .
$$

(v) the boundary of $V_{0}$ is locally a Lipschitz graph.

Proof. Fix $\rho>0$ small, and for $0<h \leq 1$ set

$$
E_{\rho}(h)=\left\{x \in h \mathbb{Z}^{d}:|x|>\rho\right\} .
$$

Extend each $u_{h}$ as 0 outside $V_{h}$. From Proposition 5.2 and definition of $u_{h}$, there exists a constant $C_{\rho}=C(\rho, d)$ independent of $h$ such that

$$
\left|u_{h}(x)-u_{h}(y)\right| \leq C_{\rho}|x-y|, \quad x, y \in E_{\rho}(h) .
$$

For $y \in \partial V_{h}$ we have $u_{h}(y)=0$, hence for any $x \in E_{\rho}(h) \cap V_{h}$ thanks to (5.4) we obtain

$$
\left|u_{h}(x)\right| \leq C_{\rho}|x| \leq C_{\rho} C_{1},
$$

where the second inequality with a constant $C_{1}=C_{1}(d)$ follows from Lemma 4.12. Thus each $u_{h}, 0<h \leq 1$, when restricted to $E_{\rho}(h)$, is bounded uniformly in $h$ due to (5.5) and is $C_{\rho}$-Lipschitz in view of (5.4). Next, we extend each $u_{h}$ from $E_{\rho}(h)$ to a Lipschitz function on $\mathbb{R}^{d}$ having the same Lipschitz constant. Namely, for a given $h>0$ define

$$
U_{h}^{\rho}(x)=\inf _{\xi \in E_{\rho}(h)}\left(u_{h}(\xi)+C_{\rho}|x-\xi|\right), \quad x \in \mathbb{R}^{d} .
$$

This is a well-known method of Lipschitz extension, and it is not hard to verify that $U_{h}^{\rho}$ is a $C_{\rho}$-Lipschitz function on $\mathbb{R}^{d}$ (see e.g. [17, Theorem 2.3]) and coincides with $u_{h}$ on $E_{\rho}(h)$. Moreover, by construction we have that the family $\left\{U_{h}^{\rho}\right\}_{0<h \leq 1}$ is uniformly bounded and is non-negative everywhere. Observe also that for $0<h \leq 1$, $\xi \in E_{2 \rho}(h)$ and $x \in \mathrm{C}_{h}(\xi)$ (see (5.3)) by construction we have

$$
\left|u_{h}(x)-U_{h}^{\rho}(x)\right|=\left|U_{h}^{\rho}(\xi)-U_{h}^{\rho}(x)\right| \leq C_{\rho} h .
$$

By Arzelà-Ascoli there is sequence $h_{k} \rightarrow 0$ as $k \rightarrow \infty$, and $C_{\rho}$-Lipschitz function $U_{0}^{\rho}: \mathbb{R}^{d} \rightarrow \mathbb{R}$ such that $U_{h_{k}}^{\rho} \rightarrow U_{0}^{\rho}$ locally uniformly in $\mathbb{R}^{d}$. Due to (5.7) we get $u_{h_{k}} \rightarrow U_{0}^{\rho}$ locally uniformly in $E_{2 \rho}^{\square}$. Obviously $U_{0}^{\rho} \geq 0$ on $\mathbb{R}^{d}$.

For the support of $U_{0}^{\rho}$ we have

$$
\left\{x \in \mathbb{R}^{d}: U_{0}^{\rho}(x)>0\right\} \backslash \overline{B_{2 \rho}}=\left(\liminf _{k \rightarrow \infty} V_{h_{k}}^{\square}\right)^{\circ} \backslash \overline{B_{2 \rho}} .
$$

To prove this take any $x$ from the l.h.s. of (5.8). Since $U_{0}^{\rho}$ is continuous on $\mathbb{R}^{d}$ there is $r>0$ such that the closed ball $\overline{B_{r}}(x) \subset \mathbb{R}^{d} \backslash \overline{B_{2 \rho}}$ and $U_{0}^{\rho}>\varepsilon$ on $\overline{B_{r}}(x)$ for some $\varepsilon>0$. By construction we have that $U_{h_{k}}^{\rho}$ converges to $U_{0}^{\rho}$ uniformly on $\overline{B_{r}}(x)$, hence there exists $k_{0} \in \mathbb{N}$ large enough such that $U_{h_{k}}^{\rho}(x) \geq \varepsilon$ on $\overline{B_{r}}(x)$ for any integer $k>k_{0}$. But since $U_{h_{k}}^{\rho}$ agrees with $u_{h_{k}}$ on $E_{\rho}\left(h_{k}\right)$ we get $u_{h_{k}}(\xi) \geq \varepsilon$ 
for any $\xi \in h_{k} \mathbb{Z}^{d} \cap \overline{B_{r / 2}}(x)$. This implies $u_{h_{k}}(x) \geq \varepsilon$ on $\left(h_{k} \mathbb{Z}^{d} \cap \overline{B_{r / 2}}(x)\right)^{\square}$ hence $\overline{B_{r / 2}}(x) \subset V_{h_{k}}^{\square} \backslash \overline{B_{2 \rho}}$ for all $k>k_{0}$. In particular, $x$ is from the r.h.s. of (5.8).

To see the reverse inclusion fix any $x$ from the r.h.s. of (5.8). There exists $k_{0} \in \mathbb{N}$ large and $r>0$ small such that $B_{r}(x) \subset \bigcap_{k=k_{0}}^{\infty} V_{h_{k}}^{\square} \backslash \overline{B_{2 \rho}}$. We get, in particular, that $\operatorname{dist}\left(x, \partial V_{h_{k}}^{\square}\right) \geq r$ for all $k>k_{0}$. Hence, by Lemma 4.14 and definition of $u_{h_{k}}$ one gets $u_{h_{k}} \geq c_{0}>0$ on $B_{r}(x)$ with a constant $c_{0}$ uniform in $k>k_{0}$. This bound, together with (5.7) implies $U_{0}^{\rho}(x) \geq c_{0}$, accordingly $x$ lies in the l.h.s. of (5.8). This completes the proof of $(5.8)$.

We next prove that $U_{0}^{\rho}$ is harmonic on $\left\{x \in \mathbb{R}^{d}: U_{0}^{\rho}(x)>0\right\} \backslash \overline{B_{2 \rho}}$. Fix any $x \in \mathbb{R}^{d} \backslash \overline{B_{2 \rho}}$ such that $U_{0}^{\rho}(x)>0$. Since $U_{0}^{\rho}$ is continuous, there exists a closed ball $\overline{B_{2 r}}(x) \subset\left\{U_{0}^{\rho}>0\right\} \backslash \overline{B_{2 \rho}}$. Let $\varphi \in C_{0}^{\infty}\left(B_{r}(x)\right)$ be any. Since $U_{0}^{\rho}$ is continuous, to prove its harmonicity in $B_{r}(x)$ it suffices, due to Weyl's lemma, to show that $\int_{\mathbb{R}^{d}} U_{0}^{\rho} \Delta \varphi d x=0$ where $\Delta$ is the usual (continuous) Laplace operator in $\mathbb{R}^{d}$. The latter follows by

$$
\int_{\mathbb{R}^{d}} U_{0}^{\rho} \Delta \varphi d x=\lim _{k \rightarrow \infty} \int_{\mathbb{R}^{d}} U_{h_{k}}^{\rho} \Delta^{h_{k}} \varphi d x=\lim _{k \rightarrow \infty} \int_{\mathbb{R}^{d}} u_{h_{k}} \Delta^{h_{k}} \varphi d x=\lim _{k \rightarrow \infty} \int_{\mathbb{R}^{d}} \Delta^{h_{k}} u_{h_{k}} \varphi d x=0,
$$

where the first equality comes from definition of $U_{0}^{\rho}$ and smoothness of $\varphi$, the second is in view of (5.7), the third one follows from discrete integration by parts. The last equality is a consequence of the fact that for large enough $k_{0} \in \mathbb{N}$ due to (5.8) one has $B_{2 r}(x) \subset V_{h_{k}}^{\square} \backslash \overline{B_{2 \rho}}$ for all $k>k_{0}$, which, in particular, implies that $\Delta^{h_{k}} u_{h_{k}}=0$ on $B_{r}(x)$ for all $k>k_{0}$.

Taking the parameter $\rho \rightarrow 0$ and applying a diagonal argument we conclude that there is a non-negative function $u_{0} \in C\left(\mathbb{R}^{d} \backslash\{0\}\right)$, and a sequence $h_{k} \rightarrow 0$ such that

(a) $u_{h_{k}} \rightarrow u_{0}$ locally uniformly outside any neighbourhood of 0 ,

(b) $\operatorname{supp} u_{0}=\liminf _{k \rightarrow \infty} V_{h_{k}}^{\square}$,

(c) $\Delta u_{0}=0$ in $\left\{x \in \mathbb{R}^{d} \backslash\{0\}: u_{0}(x)>0\right\}$,

(d) $u_{0}$ is Lipschitz continuous outside any neighbourhood of the origin, where Lipschitz norm depends on the neighbourhood.

These properties give us parts (i) and (iii) of the theorem, as well as the claim of (ii) except at the origin. To finish the proof of part (ii) it remains to study the Laplacian of $u_{0}$ at 0 . To this end, let $\Phi_{h}$ be the fundamental solution for $\Delta^{h}$ in $h \mathbb{Z}^{d}$, i.e. $\Delta^{h} \Phi_{h}=-h^{d} \delta_{0}$. Here as well, following the convention above, suppose that $\Phi_{h}$ is extended to $\mathbb{R}^{d}$ so that to preserve its $\Delta^{h}$-Laplacian. It is well-known (see [22]) that $\Phi_{h} \rightarrow \Phi_{0}$ as $h \rightarrow 0$ locally uniformly in $\mathbb{R}^{d} \backslash\{0\}$, where $\Phi_{0}$ is the fundamental solution to continuous Laplacian. Observe, that in view of (b) and Lemma 4.12 there is $r>0$ small such that the ball $\bar{B}_{r} \subset \operatorname{supp} u_{0}$. Using this and the definition of $u_{h_{k}}$ we have

$$
\Delta^{h_{k}}\left(u_{h_{k}}-\Phi_{h_{k}}\right)=0 \text { in } B_{r} .
$$

But since both $u_{h_{k}}$ and $\Phi_{h_{k}}$ converge locally uniformly away from 0 to continuous functions, then the difference $\left|u_{h_{k}}-\Phi_{h_{k}}\right| \leq C$ on $\partial B_{r}$ for all $k \in \mathbb{N}$ where $C>0$ is some large constant. By DMP we get that $\left|u_{h_{k}}-\Phi_{h_{k}}\right| \leq C$ in $B_{r}$, hence, the limit $\left|u_{0}-\Phi_{0}\right|$ is also bounded by $C$ in $B_{r} \backslash\{0\}$. But as $\Delta\left(u_{0}-\Phi_{0}\right)=0$ in $B_{r} \backslash\{0\}$ we get that 0 is a removable singularity for $u_{0}-\Phi_{0}$, in particular $\Delta u_{0}=\Delta \Phi_{0}$ at 0 , and the proof of part (ii) is now complete. 
The proof of part (iv) of the theorem is a direct consequence of Theorem 4.5, and convergence of $u_{h_{k}}$ to $u_{0}$.

It remains to show $(\mathrm{v})$, the last assertion of the Theorem. We will show that at each $x^{(0)} \in \partial V_{0}$ there exists a double cone, with its size (opening and height) independent of $x^{(0)}$, having vertex at $x^{(0)}$ and intersecting $\partial V_{0}$ at $x^{(0)}$ only ${ }^{13}$. This clearly implies (v).

Applying the monotonicity of (iv) in directions $\left\{e_{i}\right\}_{i=1}^{d}$ we obtain $u_{0}(x)=u_{0}(-x)$ for all $x \in \mathbb{R}^{d}$. Due to this symmetry, to prove (v) it is enough to treat the part of $\partial V_{0}$ where all coordinates are non-negative. In addition, we will also assume that the point $x^{(0)}=\left(x_{1}^{(0)}, \ldots, x_{d}^{(0)}\right) \in \partial V_{0}$ satisfies $x_{d}^{(0)} \geq x_{i}^{(0)}$ for all $1 \leq i \leq d-1$. The rest of the cases are similar. Observe that due to (iii) and Lemma 4.12 there is a constant $c_{0}>0$ such that

$$
B_{c_{0}} \subset\left\{x \in \mathbb{R}^{d}: u_{0}>0\right\} .
$$

Now the choice of $x^{(0)}$ and (5.9) together imply

$$
x_{d}^{(0)} \geq \frac{1}{d} c_{0} .
$$

We first determine a certain monotonicity region for $x^{(0)}$. Let $\mathcal{V} \subset \mathbb{R}^{d}$ be a finite set of vectors containing $e_{d}$ and having the rest of its elements chosen by the following rule: for each $1 \leq i \leq d-1$

$(\star)$ if $x_{d}^{(0)} \geq 2 x_{i}^{(0)}$ then $e_{d}+e_{i}$ and $e_{d}-e_{i}$ are in $\mathcal{V}$,

$(\star \star)$ if $x_{d}^{(0)} \leq 3 x_{i}^{(0)}$ then $e_{i}$ is in $\mathcal{V}$.

It is easy to check from definition of $\mathcal{V}$ that it contains $d$ linearly independent vectors. For a given $v \in \mathcal{V}$ consider the halfspace $\mathcal{H}_{v}=\left\{x \in \mathbb{R}^{d}: x \cdot v>0\right\}$. We have the following monotonicity of $u_{0}$ in $\mathcal{H}_{v}$.

(5.11) If $X_{1}, X_{2} \in \mathcal{H}_{v}, X_{2}-X_{1}=t v$ with $t \in \mathbb{R}$, then $u_{0}\left(X_{1}\right) \geq u_{0}\left(X_{2}\right)$ iff $t \geq 0$.

Indeed, decomposing $X_{i}$ into tangential and normal components in $\mathcal{H}_{v}$, one gets $X_{i}=\tau_{0}+t_{i} v$, where $i=1,2, t_{i} \geq 0$ and $\tau_{0} \in \mathcal{H}_{v}$ is the same for both $X_{1}, X_{2}$ due to assumption. Then, $\left|X_{i}\right|^{2}=\left|\tau_{0}\right|^{2}+t_{i}^{2}|v|^{2}$, hence $\left|X_{1}\right| \leq\left|X_{2}\right|$ if and only if $\left|t_{1}\right| \leq\left|t_{2}\right|$ but since $t_{i} \geq 0$ the latter reduces to $t_{1} \leq t_{2}$. It remains to apply the monotonicity result of part (iv) to get (5.11). We thus obtain that $u_{0}$ is non-increasing in $\mathcal{H}_{v}$ in the normal direction.

Consider the cone

$$
\mathcal{C}_{0}=\bigcap_{v \in \mathcal{V}} \mathcal{H}_{v}
$$

It is easy to see that the bound (5.10) and definition of $\mathcal{V}$ imply the existence of a constant $c_{1}>0$ independent of $x^{(0)}$ such that

$$
B_{c_{1}}\left(x^{(0)}\right) \subset \mathcal{C}_{0}
$$

As a direct corollary of (5.11) the cone $\mathcal{C}$ inherits the following property. If $X_{1}, X_{2} \in \mathcal{C}_{0}$ and $X_{2}-X_{1}=t_{1} v_{1}+\ldots+t_{k} v_{k}$ where $t_{i} \geq 0, v_{i} \in \mathcal{V}$ for all $1 \leq i \leq k$, and $k$ is the cardinality of $\mathcal{V}$, then

$$
u_{0}\left(X_{1}\right) \geq u_{0}\left(X_{2}\right) .
$$

\footnotetext{
${ }^{13}$ Observe that $\partial V_{0}$ is the 0-level set of $u_{0}$ which is a Lipschitz function on compact subsets away from the origin, and in particular in a neighbourhood of $\partial V_{0}$. But this fact alone is not enough to conclude that $\partial V_{0}$ is Lipschitz, see for instance [3].
} 
Along with $\mathcal{C}_{0}$ consider as well the cone generated by $\mathcal{V}$

$$
\mathcal{C}_{V}=\left\{t_{1} v_{1}+\ldots+t_{k} v_{k}: t_{i} \geq 0, v_{i} \in \mathcal{V}, \text { for all } 1 \leq i \leq k \text { and } k=|\mathcal{V}|\right\} .
$$

Since $\mathcal{V}$ has $d$ linearly independent vectors, the cone $\mathcal{C}_{V}$ is $d$-dimensional. We claim that $\mathcal{C}:=\left(x^{(0)}-\mathcal{C}_{V}\right) \cap \mathcal{C}_{0}$ is the sought cone, for which we need to show that the truncated cone $\mathcal{C}$ has uniform size and that $u_{0}$ is positive in the interior of $\mathcal{C}$ and is zero in the interior of $\left(x^{(0)}+\mathfrak{C}_{V}\right) \cap \mathfrak{C}_{0}$. We will only show the former claim, as the latter follows similarly.

It is clear, thanks to (5.12), that $\mathcal{C}$ is of uniform size. Now assume for contradiction, that there is $x^{*} \in \stackrel{\circ}{\mathcal{C}}$ such that $u_{0}\left(x^{*}\right)=0$. Consider the cone $\mathcal{C}^{*}=\left(x^{*}+\mathcal{C}_{V}\right) \cap \mathfrak{C}_{0}$. Let us see that

$$
u_{0}\left(x^{*}\right) \geq u_{0}(x), \forall x \in \mathcal{C}^{*} \quad \text { and } \quad x^{(0)} \text { is in the interior of } \mathcal{C}^{*} .
$$

The first assertion of (5.14) follows directly from (5.13) since $x^{*} \in \mathcal{C}_{0}$ by definition and any element $x \in \mathcal{C}^{*}$ is from $\mathcal{C}_{0}$ as well, and has the form $x=x^{*}+t_{1} v_{1}+\ldots+t_{k} v_{k}$ with all $t_{i} \geq 0$. The second one is simply a consequence of the definition of $x^{*}$.

Armed with (5.14) the proof of ( $\mathrm{v}$ ) follows readily, since we simply get that $u_{0}$ is zero in an open neighbourhood of $x^{(0)}$, which violates the condition that $x^{(0)} \in \partial V_{0}$. This contradiction finishes the proof of $(\mathrm{v})$, and the proof of the theorem is now complete.

Proof of Theorem 4.7. Assertion (a) follows from convergence result of PegdenSmart discussed in subsection 4.3 in conjunction with Theorem 4.6. The proof of part (b) follows from part (a) by the same argument as for part (v) of Theorem 5.3. The proof is complete.

5.3. Concluding remarks and open problems. We finish the paper with a few remarks and open problems which we think are interesting.

Remark 5.4. (On monotonicity) Observe that monotonicity of the odometer proved in Theorems 4.5, 4.6, 4.7, and 5.3 is not strict. Namely, we expect the odometer to be strictly monotone on its support. Such statement can be proved for the boundary sandpile model relying on harmonicity of odometer and the strong maximum principle, however for the classical Abelian sandpile model (ASM) it seems to require a more careful analysis, which we leave open. We also think that the "Boundary Abelian sandpile" described below in the text following Problem 2, should enjoy the monotonicity property of the odometer. But to actually prove that, one needs to work out the analogue of the least action principle (see subsection 4.3).

Next, we remark there are a few interesting (and not hard to prove) corollaries of the discrete monotonicity of the odometer of the ASM proved in Theorem 4.6. First, the monotonicity of the odometer function implies that the visited set of the $A S M$ with $n \in \mathbb{N}$ chips at the origin, is simply connected for any $n$, meaning that its complement in $\mathbb{Z}^{d}$ is connected as a graph. This recovers the result of Fey and Redig [12, Proposition 4.12] by a different method. Next, relying on monotonicity of the odometer, one can show that the visited set of the ASM contains a diamond (a convex hull of the points $\left\{ \pm e_{i}, i=1, \ldots, d\right\}$ restricted to $\mathbb{Z}^{d}$ ) scaled by a factor of $R \approx n^{1 / d}$, and is contained in a cube of size $R \approx n^{1 / d}$ (cf. [1, Proposition 3.7]). Of course this result is not new, and moreover the constants we can get in these embeddings are slightly worse than the ones obtained by Fey and Redig [12], and imporved later by Levine and Peres [24]. What we think is interesting here, is 
that the method is different, and seems to be of a general nature, relying mainly on the monotonicity of the odometer. Hence, similar embeddings should be possible to obtain for the boundary sandpile, or the boundary abelian sandpile, without much difficulty.

The details of this discussion will be given elsewhere.

Problem 1. Does the boundary sandpile process, for initial distributions concentrated at a point, have a scaling limit?

It is clear from the proof of Theorem 5.3 that for any sequence of scalings $\mathfrak{h}:=\left\{h_{k}\right\}_{k=1}^{\infty}$, where $h_{k} \rightarrow 0$, one may extract a subsequence $\left\{h_{k_{m}}\right\}$ such that the corresponding sequence of scaled odometers will have a limit, call it $u_{\mathfrak{h}}$, satisfying the same properties as the function $u_{0}$ of Theorem 5.3. In particular, by Theorem 5.3 (ii) we have that any such $u_{\mathfrak{h}}$ is $C^{\infty}$ on its positivity set away from the neighbourhood of the origin, and is Lipschitz continuous up to the boundary of that set. However, we do not know if scaling limits generated by different scaling sequences must coincide.

For several lattice-growth models the existence of scaling limit, and in some cases its geometry, are known; for divisible sandpile see [26], [24] and [25], for rotor-router see [26], [24], [25], and [12], and for Abelian sandpile see [28]. The internal diffusion limited aggregation (IDLA), which is a stochastic growth model, is studied in [19], [20], [5], [18]. For the IDLA in critical regime, one may consult [14]. Growth models involving continuous amounts of mass similar to divisible sandpile model, are studied in [11], [27], and [10], where the last paper considers a non-abelian growth model.

Problem 2. Assume the answer to Problem 1 is yes. Then, what would be the limiting PDE problem and what can be said about the geometry of the limiting shape? In particular, will it be convex?

It is interesting to observe, that the strong flat patterns appearing on the boundary of a sandpile as can be seen in Figure 1, might be a result of a significant increase of the role of the free boundary, rather than the PDE problem in the interior. It is remarkable that a similar geometry of the boundary as in Figure 1, in particular the flatness, arises also from the classical Abelian sandpile, see Figure 2, if one treats the boundary of the growth cluster likewise, i.e. allowing each boundary point to accumulate many particles however not exceeding the given threshold. Nevertheless, the PDE problem of the Abelian sandpile in the set of visited sites, is very different from that of the boundary sandpile considered here. It seems very interesting to understand the emergence of the flatness on the boundary rigorously.

Yet another surprising property of the boundary sandpile is the discontinuity of its geometry under small perturbations of the initial mass distribution, and a certain tendency of the sandpile dynamics towards generating convex sets (see Figure 3). A rigorous explanation of these phenomena seems to be a very interesting problem.

Problem 3. Is there a boundary sandpile type process, leading to that the total mass of the system is being redistributed onto the combinatorial free boundary (possibly with a different background rules) which has a sphere as its scaling limit?

In fact, this problem does not exclude, as a possible candidate, the boundary sandpile process considered in this paper. It is apparent from numerical simulations (see Figure 1), that the shape of BS divaricates from a sphere. However, we do not have a rigorous proof of this fact. 


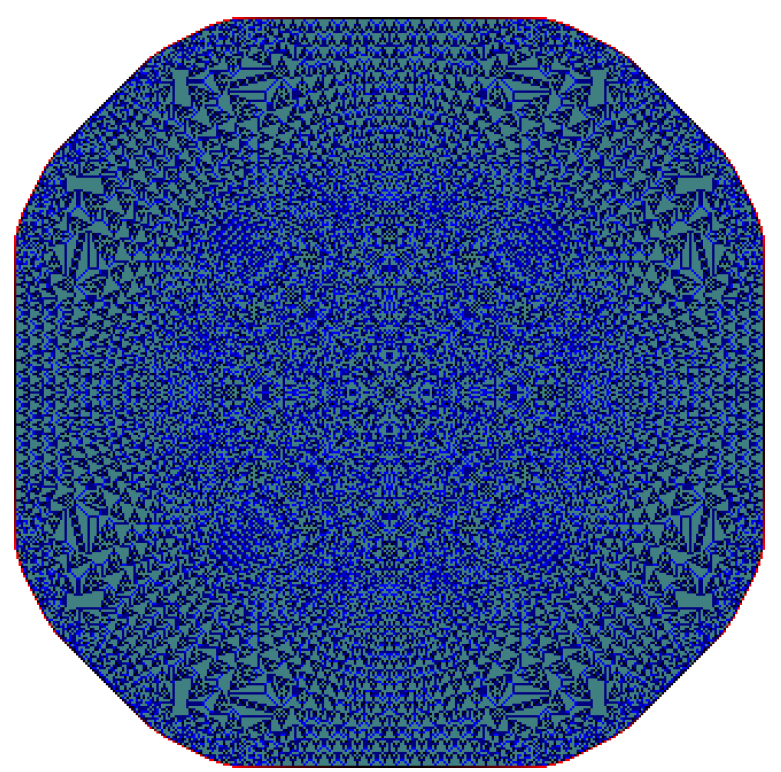

Figure 2. The classical Abelian Sandpile on $\mathbb{Z}^{2}$ obtained from one million particles at the origin and having boundary capacity equal 1000 . Lattice sites carrying 0,1,2,3 particles are coloured respectively by black, blue, dark blue, and cyan. The colouring scheme of the boundary is the same as in Figure 1.
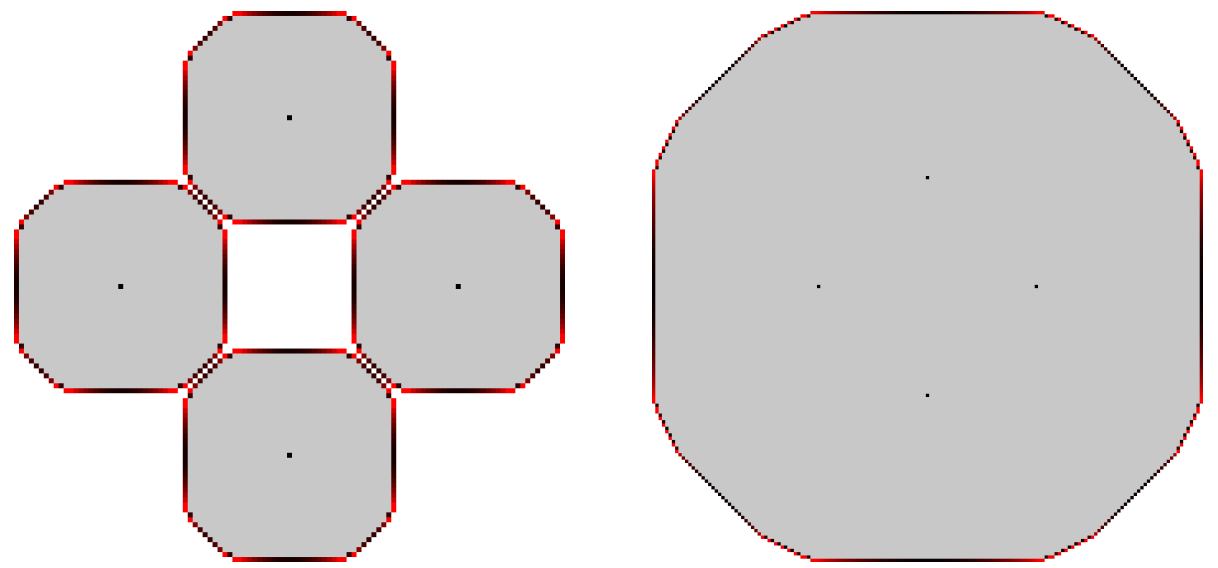

FIGURE 3. The left image is a BS with initial distribution concentrated at points $( \pm 34,0)$ and $(0, \pm 34)$ on $\mathbb{Z}^{2}$, all having mass 40000 , and the boundary capacity is set to 400 . The clusters barely survive intersection by one lattice site. On the right, is a BS having capacity 400, where mass 40000 is concentrated on each of the sites $( \pm 33,0)$ and $(0, \pm 33)$. The colouring scheme is identical to that of Figure 1.

Months after the submission of this paper, the current authors found a new sandpile redistribution rule [1], which partially answers the quest of Problem 3, at least for single sources. The relevance of Problem 3, however, remains in place, as there is no reason to believe that there does not exist a more natural rule than the one obtained in [1]. 


\section{REFERENCES}

[1] Aleksanyan, H., Shahgholian, H.: Perturbed divisible sandpiles and quadrature surfaces. preprint arXiv:1703.07568 (2017)

[2] Alexandrov, A.D.: A characteristic property of spheres, Ann. Mat. Pura Appl. (4) 58, 303-315 (1962)

[3] Alberti, G., Bianchini, S., Crippa, G.: Structure of level sets and Sard-type properties of Lipschitz maps, Ann. Sc. Norm. Super. Pisa Cl. Sci. (5), 12(4), 863-902 (2013)

[4] Alt, H. W., and Caffarelli, L. A.: Existence and regularity for a minimum problem with free boundary, J. Reine Angew. Math. 325, 105-144 (1981)

[5] Asselah, A., Gaudillière, A., From logarithmic to subdiffusive polynomial fluctuations for internal DLA and related growth models, Ann. Probab. 41(3A), 1115-1159 (2013)

[6] Bak, P., Tang, C., Wiesenfeld, K.: Self-organized criticality: An explanation of the $1 / f$ noise,Phys. Rev. A (3) 38, 364-374 (1988)

[7] Björner, A., Lovász, L., Shor, P.: Chip-firing games on graphs, European J. Combin. 4(12), 283-291 (1991)

[8] Ebenfelt, P., et al. (eds.): Quadrature Domains and Their Applications. The Harold S. Shapiro Anniversary Volume. Birkhäuser, Basel (2005)

[9] Fey, A., Levine, L., Peres, Y.: Growth rates and explosions in sandpiles, J. Stat. Phys. 138, 143-159 (2010)

[10] Fey-den Boer, A., Liu, H.: Limiting shapes for a nonabelian sandpile growth model and related cellular automata, J. Cell. Autom. 6, 353-383 (2011)

[11] Frómeta, S., Jara, M.: Scaling limit for a long-range divisible sandpile. preprint at arXiv:1507.03624 (2015)

[12] Fey, A., Redig, F.: Limiting shapes for deterministic centrally seeded growth models. J. Statist. Phys. 130(3), 579-597 (2008)

[13] Fukai, Y., Uchiyama, K.: Potential kernel for two-dimensional random walk, Ann. Probab. 24(4), 1979-1992 (1996)

[14] Gravner, J., Quastel, J.: Internal DLA and the Stefan problem, Ann. Probab. 28(4), 1528-1562 (2000)

[15] Gustafsson, B.: Direct and inverse balayage-some new developments in classical potential theory. Proceedings of the Second World Congress of Nonlinear Analysts, Part 5 (Athens, 1996) Nonlinear Anal. 30 (1997), no. 5, 2557-2565

[16] Gustafsson, B., Shahgholian, H.: Existence and geometric properties of solutions of a free boundary problem in potential theory, J. Reine Angew. Math. 473, 137-179 (1996)

[17] Heinonen, J.: Lectures on Lipschitz analysis. University of Jyväskylä (2005)

[18] Jerison, D., Levine, L., Sheffield, S.: Logarithmic fluctuations for internal DLA, J. Amer. Math. Soc. 25, 271-301 (2012)

[19] Lawler, G., Bramson, M., Griffeath, D.: Internal diffusion limited aggregation. Ann. Probab. 20(4), 2117-2140 (1992)

[20] Lawler, G.: Subdiffusive fluctuations for internal diffusion limited aggregation. Ann. Probab. 23(1), 71-86 (1995)

[21] Lawler, G.: Intersections of Random Walks, (Probability and Its Applications) Birkhäuser 1996

[22] Lawler, G., Limic, V.: Random Walk: A modern introduction, Cambridge Studies in Advanced Mathematics 2010

[23] Levine, L., Pegden, W., Smart, C.K.: Apollonian structure in the Abelian sandpile, Geom. Funct. Anal. 26(1) 306-336 (2016)

[24] Levine, L., Peres, Y.: Strong spherical asymptotics for rotor-router aggregation and the divisible sandpile, Potential Anal. 30(1), pp.1-27 (2009)

[25] Levine, L., Peres, Y.: Scaling limits for internal aggregation models with multiple sources, J. Anal. Math. 111(1), 151-219 (2010)

[26] Levine, L.: Limit Theorems for Internal Aggregation Models, PhD thesis, University of California Berkley (2007)

[27] Lucas, C.: The limiting shape for drifted internal diffusion limited aggregation is a true heat ball, Probab. Theory Relat. Fields 159, 197-235 (2014)

[28] Pegden, W., Smart, C. K.: Convergence of the abelian sandpile, Duke Math. J. 162(4), 627-642 (2013) 
[29] Serrin, J.: A symmetry problem in potential theory, Arch. Ration. Mech. Anal. 43, 304-318 (1971)

[30] Uchiyama, K.: Green's functions for random walks on $\mathbb{Z}^{N}$, Proc. Lond. Math. Soc. 77(1), 215-240 (1998)

[31] Zidarov, D.: Inverse Gravimetric Problem in Geoprospecting and Geodesy. Developments in solid earth geophysics, Elsevier (1990)

Department of Mathematics, KTH Royal Institute of Technology, SE-100 44 StockHOLM, SWEDEN

E-mail address: hayk.aleksanyan@gmail.com

Department of Mathematics, KTH Royal Institute of Technology, SE-100 44 StockHOLM, SWEDEN

E-mail address: henriksh@math.kth.se 